\title{
Article
}

\section{Looking, seeing and believing in autism: Eye movements reveal how subtle cognitive processing differences impact in the social domain}

Benson, Valerie, Castelhano, MS, Howard, PL, Latif, N and Rayner, $\mathrm{K}$

Available at http://clok.uclan.ac.uk/28048/

Benson, Valerie ORCID: 0000-0002-0351-4563, Castelhano, MS, Howard, PL, Latif, $N$ and Rayner, $K$ (2016) Looking, seeing and believing in autism: Eye movements reveal how subtle cognitive processing differences impact in the social domain. Autism research : official journal of the International Society for Autism Research, 9 (8). pp. 879-887. ISSN 1939-3792

It is advisable to refer to the publisher's version if you intend to cite from the work. http://dx.doi.org/10.1002/aur.1580

For more information about UCLan's research in this area go to http://www.uclan.ac.uk/researchgroups/ and search for <name of research Group>.

For information about Research generally at UCLan please go to http://www.uclan.ac.uk/research/

All outputs in CLoK are protected by Intellectual Property Rights law, including Copyright law. Copyright, IPR and Moral Rights for the works on this site are retained by the individual authors and/or other copyright owners. Terms and conditions for use of this material are defined in the policies page. 
Autism Research

\section{Looking, Seeing and Believing in Autism: Eye Movements reveal how subtle cognitive processing differences impact in the social domain.}

\begin{tabular}{|r|l|}
\hline Journal: & Autism Research \\
\hline Manuscript ID & AUR-15-0133.R2 \\
\hline Wiley - Manuscript type: & Research Article \\
\hline Complete List of Authors: & $\begin{array}{l}\text { Benson, Valerie; University of Southampton, School of Psychology } \\
\text { Castelhano, Monica; Queens University, Psychology } \\
\text { Howard, Phillipa; University of Southampton, Psychology } \\
\text { Latif, Nida; Queens University, Psychology } \\
\text { Rayner, Keith; University of California, San Diego, Psychology }\end{array}$ \\
\hline Keywords: & $\begin{array}{l}\text { Developmental Psychology, Neuropsychology, Autism, Eye movements, } \\
\text { cognitive processing differences }\end{array}$ \\
\hline &
\end{tabular}




\title{
Page 1 of 116

Looking, Seeing and Believing in Autism: Eye Movements reveal how subtle cognitive processing differences impact in the social domain.

\author{
Valerie Benson ${ }^{1 *}$, Monica S. Castelhano ${ }^{2}$, Philippa L. Howard ${ }^{1}$, Nida Latif $^{2}$ \& Keith Rayner $^{3}$ \\ ${ }^{1}$ AU Psychology, Shackleton Building, Highfield Campus, University of Southampton, UK. \\ ${ }^{2}$ Queen's University, Kingston, ON, Canada \\ ${ }^{3}$ University of California, San Diego, USA \\ *Correspondence to: vb1@soton.ac.uk \\ Telephone: +44 (0) 2380594596
}




\section{Lay Abstract}

Adults with High Functioning Autism (ASD) viewed scenes with people in them, whilst having their eye movements recorded. The task was to indicate, using a button press, whether the pictures were normal, or in some way weird or odd. Oddities in the pictures were categorized as violations of either perceptual or social norms. Compared to a Typically Developed (TD) control group, the ASD participants were equally able to categorise the scenes as odd or normal, but they took longer to respond. The eye movement patterns showed that the ASD group made more fixations and revisits to the target areas in the odd scenes compared to the TD group. Additionally, when the ASD group first fixated the target areas in the scenes, they failed to initially detect the social oddities. These two findings have clear implications for processing difficulties in ASD for the social domain, where it is important to detect social cues on-line, and where there is little opportunity to go back and recheck possible cues in fast dynamic interactions. 
Scientific Abstract

\begin{abstract}
Autistic Spectrum Disorder (ASD) and Typically Developed (TD) adult participants had to decide whether scenes were 'odd', 'unusual' or 'weird'. All scenes contained people, with 'oddness' operationally defined as either perceptual or social. There were no group differences in accuracy, but the ASD group were slower overall to respond manually. Eye movements revealed that the ASD group made more fixations overall and more re-fixations into the target regions of the pictures, for both conditions. Importantly, as indexed by the first fixation duration the ASD group failed to 'pick up' immediately on what was 'odd' for the social violations. The propensity to 'go back' and re-fixate targets, coupled with a failure to initially detect social violations, has obvious significance for ASD in fast dynamic social communication.
\end{abstract}

Key phrases: Autism Spectrum Disorder, Eye movements, On-line cognitive processing, Social and Perceptual Oddities. 
Looking, Seeing and Believing in Autism: Eye Movements reveal how subtle cognitive processing differences impact in the social domain.

Autism spectrum disorders (ASD) are lifelong neuro-developmental conditions characterized by repetitive behaviours and impairments in interaction and communication (American Psychiatric Association, 2013). Incidence rates are rising across the globe with the prevalence rate in the UK approximately 10 per 1000 (1\%) individuals (Brugha et al., 2011) and the UK National Audit office recently called for more research into adults with ASD (Clark et al., 2009).

High functioning adults with ASD are similar to typically developed (TD) adults in terms of performance on pencil and paper and IQ tests, however in the social domain evidence suggests an inability to process social information in a typical fashion. Eye movement studies report unusual gaze behaviour for faces appearing on their own (Dalton et al., 2005), in social scenes (Riby \& Hancock, 2009) or in dynamic movie clips (Klin \& Jones, 2006; Speer et al., 2007). Research consistently reports a lack of spontaneous gaze fixation towards the eyes (Jones et al., 2008; Klin et al., 2002a; Pelphrey et al., 2002). However, where one study reports impairments, say for face processing (Klin et al., 2002b), another study does not (Fletcher-Watson et al., 2009). Moreover, lack of attentional modulation for social stimuli (Bird et al., 2006) may be overridden, as when cued, attention may be allocated to faces in a more 'typical' manner (BarHaim et al., 2006).

A recent scene perception study (Louwerse et al., 2014) reported that abnormal autonomic responses in ASD are unlikely to underpin social processing impairments. It is interesting then to ask whether impairments in the cognitive domain in ASD (Happé \& Frith, 2006; Minshew et al., 1997; Russell, 1997) could account for impairments in the social domain? 
Current cognitive theories of ASD include, among others, Weak Central Coherence (WCC) theory (Frith \& Happé, 1994; Happé \& Frith, 2006) which proposes that a reduced sensitivity to global context in favour of focussing on details, results in integration difficulties in ASD. Executive function theory (Russell, 1997), proposes that ASD are impaired at switching their focus of attention, and may engage is perseverative behaviour. Alternatively, the Complex Information Processing theory (Minshew \& Goldstein, 1998) proposes that individuals with ASD have impairments in processing complex information with intact processing for tasks that require simple (rule based) processing. Finally, the Extreme Male Brain (EMB) theory (Baron-Cohen, 2002) posits that individuals with ASD have above average levels of systemizing, that contribute to the non-social characteristics of the disorder, coupled with below average levels of empathizing, which results in atypical social functioning. These contemporary theories of ASD can, for different reasons, offer some account of ineffective social functioning in ASD. However, to date very few investigations have examined processing differences as these occur on-line in ASD.

Our studies investigating cognitive processing in adults with ASD (Au-Yeung et al., 2011; Au-Yeung et al., 2013; Benson et al., 2012; Benson et al., 2009; Benson \& FletcherWatson, 2011) have found subtle on-line processing differences, as revealed by eye movements, for a range of tasks that are more in line with Minshew and Goldsteins (1998) theory, than with any other. For example, there are more similarities than differences in eye movements between TD and ASD groups for simple rule based tasks in the attentional (Kuhn et al., 2010) and the social domains (Benson \& Fletcher-Watson, 2011). Whereas for more complex abstract reasoning tasks where there is ambiguity with relation to target items (Au-Yeung et al., 2013), or where value judgements rely on top down knowledge (Benson et al., 2012), there are early 
processing differences indicating that initial orienting to, and immediate detection of weird target items in scenes is absent in ASD. Moreover, the time to respond and to locate the weird target items was modulated by whether or not the pictures had people in them (Benson et al., 2012). These differences have potential for impact on everyday activities, since the time course for detection of socially relevant information is crucial for successful everyday interactions.

The aim of the present study was to examine whether increased times to find and initially detect an unusual target in a scene would be modulated by whether the violation that rendered the target unusual or odd could be categorised as social or perceptual. We employed a similar paradigm to that used in a previously reported study (Rayner et al., 2009) where single scenes, all with people in them, were presented in isolation and the task was to indicate whether each picture was normal or in some way weird unusual or odd.

\section{Insert Figure 1. about here}

Social oddities in the pictures were operationally defined as violations of social norms e.g. someone in the picture throwing a baby in the air. Access to top down knowledge is required in that condition to inform a subjective value judgement as to whether a social violation is present in the picture. No value judgement as to whether a violation infringes some implicit or explicit social rule is needed for the perceptual oddities in this study, which are operationally defined as physical violations e.g. someone in the picture has a limb positioned back to front. See supplementary information for Materials. Figure 1 shows an example of the stimuli.

\section{Method}

\section{Participants}


ASD participants ( $n=24$ age $18-52$ years, 20 males), with a formal diagnosis of either Autistic Disorder $(n=3)$ or Asperger's Syndrome were recruited from local charitable organisations. TD participants $(n=24$, age $20-52$ years, 20 males) were volunteers from the local community. All participants were paid and had normal or corrected to normal vision. Each ASD participant completed module 4 of the Autism Diagnostic Observation Scale 2 (Lord et al., 2012) and five (3 males and 2 females with a diagnosis of Asperger's Syndrome) failed to meet the Autism Spectrum criteria. To ensure maximum homogeneity in our study those five participants were excluded from the analyses, resulting in a final sample of 19 ASD participants. Both groups completed the Autism-spectrum Quotient (AQ; Baron-Cohen et al., 2001) and the ASD participants reported a higher number of autistic traits $t(41)=8.04, p<.001$ (TD $M=$ 15.42, $S D=7.55$; ASD $M=35.32, S D=8.45)$. The two groups did not differ in age $t(41)=$ $0.31, p=.759(\mathrm{TD} M=30.29, S D=10.20 ;$ ASD $M=31.26, S D=10.29)$, performance IQ $t(41)$ $=0.21, p=.832(\mathrm{TD} M=111.79, S D=12.01 ; \mathrm{ASD} M=110.95, S D=13.84)$, verbal IQ $t(41)=$ $1.21, p=.234(\mathrm{TD} M=116.96, S D=10.65, \mathrm{ASD} M=112.26, S D=14.84)$, or full scale IQ $t$ $(46)=0.87, p=.392(\mathrm{TD} M=116.04, S D=9.98, \mathrm{ASD} M=112.95, S D=13.48)$, as measured by two performance (Block Design and Matrix Reasoning) and verbal components (Vocabulary and Similarity) of the Weschler Abbrevated Scale of Intelligence (The Psychological Corporation, 1999).

\section{Eye movement recording}

Eye movements were sampled at $1000 \mathrm{~Hz}$ using an Eyelink tracker with spatial accuracy of less than $1 / 4^{\text {th }}$ degree (S.R. Research Ltd, Canada). The start and end of saccades were detected automatically using a default velocity criterion of 30degrees per second using the 
Eyelink software. See Stampe (1993) for an in depth technical description of how the software detects saccades and fixations. A chin rest and forehead support, were used to stabilize head position. Viewing was binocular but data were analysed for the right eye only. Individual participants were calibrated using a nine point matrix that covered the dimensions of the screen (1024 x 768 pixels), with each point fixated sequentially, followed by a validation procedure to ensure that fixation was within 0.5 degrees of each calibration point. Calibration was checked using a validation procedure prior to each trial, whereby participants fixated five dots, presented at five different locations that covered the dimensions of the screen and recalibration was performed if the eyes were off centre for any of these dots.

\section{Materials}

In total, 96 full-colour photographs were used as stimuli, all of which contained people. In both the perceptual and social oddity conditions there were 48 pictures (24 weird and 24 normal). Perceptual oddities in the pictures violated a physical norm e.g. someone in the picture has a limb positioned back to front, or a vehicle was in an unexpected position e.g midway through the upper wall of a building. Social oddities in the pictures violated a social norm e.g. someone in the picture throwing a baby in the air, or someone with a cabbage on a lead. A small number of the pictures were from a previous study (Benson et al., 2012) and the remainder were collected from the internet and included either a perceptual or social oddity. Adobe Photoshop was used to prepare a normal version of each scene in which the oddity was replaced with either the background elements or with another suitable object. The scenes typically had a focal object or a central group of objects. Figure 1 shows an example of a normal and a socially odd scene, and below those examples, a normal and a perceptually odd scene. The target regions for these 
scenes are outlined in black - but these black outlines were not visible during the presentation of the scenes in the experiment.

A norming study was conducted to verify the validity of the images used to examine the processing of social and perceptual violations. Twenty undergraduates from Queen's university participated ( $M$ age: 19.8years, $S D: 1.71,10$ Females) with five participants in each of the four conditions (counterbalanced conditions: weird/normal and right/left button response). Stimuli were displayed on a 2 inch CRT monitor with a refresh rate of $100 \mathrm{~Hz}$ and the experiment was run using E-Prime software. Participants were presented with a fixation cross for $500 \mathrm{~ms}$ followed by the presentation of the image for 20 seconds or until a keyboard "Y' (yes) or "N" (N) was made. Participants had to indicate as quickly as possible whether the presented image was "odd", "weird" or "unusual" in some way. The proportion of identified images indicated that on average, the social normal $(M=0.86, S D=0.15)$ and social weird $(M=0.84, S D=0.17)$, perceptual normal $(M=0.82, S D=0.17)$ and perceptual weird $(M=0.77, S D=0.19)$ images were identified correctly.

\section{Design}

For the behavioural measures of Accuracy and RT a mixed design with Picture Type (weird versus normal) and Oddity Type (social versus perceptual) as within participants variables, and Group (ASD versus TD) as a between participants variable was employed for the experiment. On condition that accuracy was equivalent for both normal and weird pictures, for the eye movement measures the main focus of interest was to compare social versus perceptual oddities in both groups. Therefore a mixed design with Oddity Type (social versus perceptual) as 
a within participants variable, and Group (ASD versus TD) as a between participants variable was employed in the eye movement analysis.

\section{Procedure}

All participants were shown a hard copy of an example picture from each condition prior to completing the task, to ensure comprehension of the task and the ability to respond appropriately. The specific instruction was 'for each of the following pictures, please indicate whether you think the picture is odd, unusual or strange in some way'.

For the task, 48 pictures were presented randomly to either the left or the right of the centre of a 19inch colour monitor, at a distance of $65 \mathrm{~cm}$ for $20 \mathrm{~s}$ or until a button press response was made. Prior to the onset of a picture, participants fixated a cross that was presented on the opposite side of the screen to where the picture was set to appear, to ensure all participants started at the same point in each trial. Once participants fixated this cross, the experimenter initiated each trial. Picture type (normal vs. weird) and, the position of the picture (left or right on the display) were counterbalanced across items. The buttons used to respond either yes or no (left or right trigger button) was counterbalanced across participants.

\section{Data preparation and analyses}

For each 'odd' picture we created an interest region around the target item and the corresponding region of the same size in the counterpart 'normal' picture. Regions of interest were created using the approximate average regions from those identified and manually selected by participants who took part in the norming experiment. 
Eyelink software was used to detect saccades and fixations using a default velocity criterion and in-house software was used to calculate the distribution of fixations falling into the target region. Five items were removed from analysis because for these scenes, participant accuracy scores were significantly below chance ${ }^{1}$. In addition, each participant was asked whether they had seen any of the scenes previously, and if so these scenes were also removed. This resulted in a loss of $2.3 \%$ of trials. Fixations below $90 \mathrm{~ms}$ and above $1200 \mathrm{~ms}$ were classed as outliers and removed; resulting in a loss of $5.13 \%$ of the fixation data.

\section{Results}

For the principal analyses we compared manual reaction times and accuracy (see Table 1), as well as a number of eye movement measures, such as, the time taken to begin fixating in the target region (ELT), the duration of the first fixation when the eyes landed in the target region (FFD) and the mean time (MFD) spent fixating in the target area (see Table. 2). Global eye movement measures for the target region, such as mean fixation duration and mean number of fixations over all trials were also analysed, Finally, baseline measures (not just target related), were calculated across the duration of the trials, to check for overall sampling differences between the ASD and TD participants (see Table 2). See supplementary information for methods and materials.

\section{Behavioural Data}

Accuracy. There was no effect of oddity type (perceptual vs. social) $F(1,41)=1.44, p=$ $.237, \eta_{\mathrm{p}}^{2}=.03$, picture type (normal or weird) $F(1,41)=0.94 p=.339, \eta_{\mathrm{p}}{ }^{2}=.02$, or group, $F(1$, $41)=.06, p=.808, \eta_{\mathrm{p}}{ }^{2}<.01$ on accuracy rates, and no interactions $\left(F_{\mathrm{s}}<2.39, p\right.$ 's $\left.>.130\right)$. At 
least in terms of accuracy, performance was the same for both groups. For means and standard deviations for behavioural data see Table 1 .

Response Time (RT). A main effect of oddity type $F(1,41)=8.16, p=.007, \eta_{\mathrm{p}}{ }^{2}=.17$, showed participants took longer to respond when presented with a perceptual oddity in comparison to a social oddity. Longer response times for perceptual oddities could reflect that these 'impossible', physical violations were so unusual that they resulted in more time spent looking at them prior to responding. See below for confirmation of that in the eye movement data. There was also a main effect of picture type $F(1,41)=24.03, p<.001, \eta_{\mathrm{p}}{ }^{2}=.37$, with participants taking longer to respond when the picture was normal in comparison to when it was weird, in line with longer reaction times for target absent trials in standard visual search tasks (Wolfe, 1998) A significant oddity type $\mathrm{x}$ picture type interaction $F(1,41)=4.22, p=.046, \eta_{\mathrm{p}}{ }^{2}$ $=.09$, demonstrated that the difference between RTs for perceptual and social pictures was reliable for odd (TD; $t$ (23) 2.62, $p=.015$, ASD; $t(18)=2.98, p=.008$ ) but not for normal pictures $(\mathrm{TD} ; t(23) 0.94, p=.355$, ASD: $t(18)=0.08, p=.935)$. A main effect of group $F(1$, $41)=4.07, p=.050, \eta_{\mathrm{p}}{ }^{2}=.09$, showed the ASD participants had longer reaction times, in line with a previous study (Benson et al., 2012$)$. There were no significant group interactions $(F \mathrm{~s}<$ 1) demonstrating both groups to be slower for normal versus odd pictures, and for perceptual vs social oddities. See Table 1 for the means. The eye movement data below reveals subtle processing differences that contribute to this overall slowed manual responding in the ASD group.

Eye Movement Data 
Having shown that all participants could distinguish between 'odd' and 'normal' pictures, normal picture trials were excluded from the eye movement analyses resulting in a 2 (group: ASD versus TD) X 2 (oddity type: social versus perceptual) design to investigate on-line processing differences between the two groups for the two types of oddities.

Baseline eye movement measures, calculated across the full length of trials indicated no group difference in mean fixation duration $F(1,41)=1.14, p=.293, \eta_{\mathrm{p}}{ }^{2}=.03$, but the ASD group made more fixations overall $F(1,41)=9.44, p=.004, \eta_{\mathrm{p}}{ }^{2}=.19$, corresponding to the increased RT's reported above. Table 2 presents a summary of all the eye movement measures.

Elapsed time to the target region (ELT). A marginal effect of oddity type $F(1,41)=3.99, p=$ $.053, \eta_{\mathrm{p}}{ }^{2}=.09$, on the time taken from the onset of each trial display to the start of the first fixation on the target region, revealed both groups took less time to fixate the target region when the oddity was perceptual in comparison to social in nature. There was no effect of group $F$ (1, $41)=0.59, p=.446, \eta_{\mathrm{p}}{ }^{2}=.01$, and no interaction $F(1,41)=0.72, p=.401, \eta_{\mathrm{p}}{ }^{2}=.02$.

First Fixation Duration (FFD) on target region. There was no effect of oddity type $F(1,41)=$ $1.84, p=.182, \eta_{\mathrm{p}}{ }^{2}=.04$ or group $F(1,41)=0.15, p=.699, \eta_{\mathrm{p}}{ }^{2}<.01$ but a significant interaction $F(1,41)=6.72, p=.013, \eta_{\mathrm{p}}{ }^{2}=.14$ (See Figure 2) revealed that FFDs were equivalent for perceptual and social oddities for TD participants $t(23)=0.86, p=.399, d=.16$, but for ASD participants FFDs for social oddities were significantly shorter, in comparison to perceptual oddities, $t(18)=2.99, p=.008, d=.65$, see Figure 2 . Given that the FFD is a measure of early processing (Liversedge \& Findlay, 2000; Rayner, 1998; Rayner, 2009). This finding indicates 
that the ASD group did not detect the social oddities as quickly as the perceptual oddities on initial fixation, whereas TD participants detected both types of oddity equally fast.

\author{
Insert Figure 2. about here
}

\begin{abstract}
First gaze duration (FGD) in the target region. The FGD is the elapsed time from when the target region is first fixated until the eye moves on to a new location. FGD includes multiple fixations in the target region reflects an overt strategy to re-examine an area. A main effect of oddity type, $F(1,41)=12.10, p=.001, \eta_{\mathrm{p}}{ }^{2}=.23$ demonstrated that both groups spent more time inspecting perceptual oddities, again highlighting the salience of the perceptual oddities in comparison to social oddities in this study. However, there was no group effect $F(1,41)=2.43$, $p=.127, \eta_{\mathrm{p}}^{2}=.06$, or interaction $F(1,41)=.08, p=.785, \eta_{\mathrm{p}}^{2}<.01$.
\end{abstract}

\begin{abstract}
Mean Fixation Count in the target region. If the ASD are slower to respond manually, but do not take longer to begin inspecting the target region, then extra time to respond might reflect more fixations in the target region. A main effect of oddity type $F(1,41)=64.27, p<.001, \eta_{\mathrm{p}}{ }^{2}=$ .61 , demonstrated more fixations in the target region when the oddity was perceptual, rather than social. A main effect of group $F(1,41)=6.99, p=.012, \eta_{\mathrm{p}}{ }^{2}=.15$ showed that the ASD group made more fixations than the TD group in the target regions, for both types of oddities. However no interaction was observed $F(1,41)=0.77, p=.386, \eta_{\mathrm{p}}{ }^{2}=.02$ indicating that the ASD group made more fixations in the target regions for both types of oddities.
\end{abstract}


Mean Fixation Duration in the target region. Longer mean fixation durations indicate that more processing has been done during the task. A main effect of oddity type $F(1,41)=8.22, p=$ $.007, \eta_{\mathrm{p}}{ }^{2}=.17$ showed significantly longer fixations for social, compared to perceptual oddity target regions. This suggests that processing social oddities was more effortful, and since there was no main effect of group $F(1,41)=0.34, p=.562, \eta_{\mathrm{p}}{ }^{2}<.01$, and no interaction $F(1,41)=$ $1.12, p=.297, \eta_{\mathrm{p}}{ }^{2}=.03$, both groups on average fixated the social oddity target region for longer than the perceptual target region. Thus, while the perceptual oddities attracted more fixations overall, individual fixations were longer for the social target region.

This interesting juxtaposition, although unrelated to the main aim of this study, suggests that difficulty associated with processing each oddity type manifests in different eye movement patterns: one reflecting an overt strategy (fixation count) to re-inspect the target area, and one reflecting more in depth on-line cognitive processing (fixation duration) on the target areas.

\footnotetext{
Mean Number of Entries to the target region. Since there were no group differences in FGD, and no increased MFD, both reported above, the delayed RT in ASD might reflect repeated visits to inspect the target region?

A main effect of oddity type was found $F(1,41)=9.83, p=.003, \eta_{\mathrm{p}}{ }^{2}=.19$, with the target region entered more often for perceptual oddities in comparison to social oddities. In addition, a main effect of group was found $F(1,41)=5.72, p=.021, \eta_{\mathrm{p}}{ }^{2}=.12$, with ASD participants entering the target regions significantly more than the TD group, before making a response. There was no interaction $F(1,41)=0.13, p=.717, \eta_{\mathrm{p}}{ }^{2}<.01$.
} 
Mean Total Time in the target region. A higher mean total time means that more attention to, and processing of, information in that region was carried out overall. This measure should complement the measures reported above - namely, that if there are more fixations overall in ASD then the Total Time spent fixating in the target region should be longer in ASD.

A main effect of oddity type $F(1,41)=34.89, p<.001, \eta_{\mathrm{p}}{ }^{2}=.46$, showed perceptual oddities received more overall processing time, in line with the previous measures for the perceptual oddities. In addition, a main effect of group $F(1,41)=6.41, p=.015, \eta_{\mathrm{p}}{ }^{2}=.14$ revealed that the ASD participants spent a longer period of time inspecting the target regions for both perceptual and social oddities. There was no interaction $F(1,41)=0.51, p=.481, \eta_{\mathrm{p}}{ }^{2}=.01$.

As above, this finding reflects a greater number of entries to the target region overall by the ASD group prior to making their decision as to whether the picture under inspection was weird, odd or unusual, irrespective of the nature of the violation (perceptual or social).

\section{Discussion}

A principal aim of the study was to investigate whether increased times in ASD to find and initially detect an unusual target in a scene containing people would be modulated by whether the violation could be categorised as social or perceptual.

Accuracy rates were equivalent and high for both groups, but the ASD group took longer to respond manually. It was hypothesised that the eye movement data would reveal the cause of this delayed response in ASD, since eye movements reflect how information is processed on-line (Liversedge \& Findlay, 2000; Rayner, 1998; 2009). The data showed that our ASD group did not take longer to find the target regions, but that they scanned the scenes more prior to responding manually, as shown by an increased number of fixations throughout each scene presentation. 
Furthermore, these increased fixations were not random, but were made to the target regions of both social and perceptually odd pictures. Thus, increased fixations reflect a propensity to reinspect the target regions prior to making a response in ASD, regardless as to the type of oddity in the scenes. It should be noted here that the oddity detection task in the current study provides the ASD participants with structure that is not typically available in real-life. As such, the task is not implicit and the knowledge that oddities might be present could also be a factor that drives the repeated scanning in ASD.

The eye movement patterns also showed that our ASD group failed to immediately detect social oddities, thus looking at but not seeing them. An obvious question from this finding is whether the failure to quickly spot subtle social oddities in ASD reflects an inability to react or respond appropriately to social cues in everyday communication? This one impairment in itself could potentially account for the significant problems experienced in dynamic social interactions in this high functioning population. For example, a failure to immediately spot social cues would result in a failure to react or respond to such cues thus resulting in an inability to both keep track of what is going on in social interactions, and a failure to engage appropriately or effectively with other people.

If social oddities are more complex to detect in ASD, then the Complex Information Processing theory (Minshew \& Goldstein, 1998) might explain the failure to detect the social oddity on first fixation. This lack of immediate detection could reflect the less obvious and more 'inferential' nature of the social oddities, which required more complex reasoning, at least in the ASD group, for detection. It is important to note that social rules are often implicit, sometimes ambiguous, and cannot always be understood by following a script or flow chart as in the case for logical rule based tasks. At a neurobiological level, it has been postulated that that 
impairments in cognitive processing in ASD reflect slow information transmission rates for high level cognitive tasks, resulting from underconnectivity between frontal-posterior cortical regions (Just et al., 2013; Just et al., 2012; Minshew \& Williams, 2007). Since underconnectivity reduces the rate of inter-region information transfer, it could be that in social communication tasks that rely on the fast detection of implicit, often ambiguous cues, a failure to be able to transmit information across different cortical areas quickly, might mean that these cues are missed, rendering inappropriate responses in ASD.

The findings of repeated scanning of the target areas, coupled with an inability to immediately recognise social violations upon initial fixation, reflect how eye movements can reveal subtle processing differences in ASD. In everyday social communication in the real world there is no opportunity to 'go back' to check if one has missed an important cue. Social processing in such circumstances must be carried out on-line, and our eye movement data clearly show that ASD are unable to do this quickly. The strategy of re-checking their interpretation, and thus not believing what they have seen, before responding, may be related to cortical underconnectivity in ASD. In everyday terms, these findings have obvious consequences for fast dynamic social interactions, and offer an account as to why high functioning adults with ASD experience difficulty in the social domain. 


\section{Acknowledgments}

This work was supported by a grant awarded to the second author from the Natural Sciences and Engineering Research Council of Canada, the Canadian Foundation for Innovation, the Ontario Ministry of Research and Innovation, and Queen's University Senate Advisory Research Committee, and an Experimental Psychology Society study visit grant awarded to the third author. 


\section{References}

American Psychiatric Association. (2013). Diagnostic and statistical manual of mental disorders (5th ed.). Arlington, VA: American Psychiatric Publishing.

Au-Yeung, S. K., Benson, V., Castelhano, M. S., Rayner, K. (2011). Eye movement sequences during simple versus complex information processing of scenes in autism spectrum disorder. Autism Research and treatment, 2011.

Au-Yeung, S. K., Kaakinen, J. K., Benson, V. (2013). Cognitive perspective taking during scene perception in autism spectrum disorder: evidence from eye movements. Autism Research, 7, 84-93.

Bar-Haim, Y., Shulman, C., Lamy, D., \& Reuveni, A. (2006). Attention to eyes and mouth in high-functioning children with autism, Journal of Autism and Developmental Disorders, $36,131-137$.

Baron-Cohen, S. (2002). The extreme male brain theory of autism. Trends in Cognitive Science, $6,248-254$.

Baron-Cohen, S., Wheelwright, S., Skinner, R., Martin, J., \& Clubley, E. (2001). The AutismSpectrum Quotient (AQ): Evidence from Asperger syndrome/high-functioning autism, males and females, scientists and mathematicians. Journal of Autism and Developmental Disorders, 31(1), 5-17.

Benson, V., \& Fletcher-Watson, S. (2012). Eye Movements in Autism. In: Liversedge, S. P., Gilchrist, I. D., Everling, S., editors. The Oxford Handbook of Eye Movements, Oxford UK: Oxford University Press, chap 39.

Benson, V., Castelhano, M. S., Au-Yeung, S. K., \& Rayner K. (2012). Eye movements reveal no immediate "WOW" ("which one's weird") effect in autism spectrum disorder, Quarterly 
Journal of Experimental Psychology, 65, 1139-1150.

Benson, V., Piper, J., \& Fletcher-Watson, S. (2009). Atypical saccadic sampling in autistic spectrum disorder, Neuropsychologia, 47, 1178-1182, (2009).

Bird, G., Catmur, C., Silani, G., Frith, C., \& Frith, U. (2006). Attention does not modulate neural responses to social stimuli in autism spectrum disorders. Neuroimaging, 15, 1614-1624.

Brugha, T. S., McManus, S., Bankart, J., Scott, F., Purdon S., et al. (2011). Epidemiology of autism spectrum disorders in adults in the community in England. Archives of Genereal Psychiatry, 68, 459-65.

Clark, F., Scharaschkin, A., Xu, D. (2009). Supporting autism through adulthood, National Audit Office.

Dalton, K. M., Nacewicz, B. M., Johnstone, T., Schaefer, H. S., Gernsbacher, M. A., Goldsmith, H. H., Alexander, A. L., \& Davidson, R. J. (2005). Gaze fixation and the neural circuitry of face processing in autism. Nature Neuroscience, 8, 519-526.

Fletcher-Watson, S., Leekam, S. R., Benson, V., Frank, M. C., \& Findlay, J. M. (2009). Eyemovements reveal attention to social information in autism spectrum disorder. Neuropsychologia, 47, 248-257.

Fletcher-Watson., Leekam, S.R., Benson, V., Frank, M.C., \& Findlay, J.M. (2009). Eyemovements reveal attention to social information in autism spectrum disorder. Neuropsychologia, 47, 248-257.

Frith, U., \& Happé, F. (1994). Autism; Beyond the theory of mind. Cognition, 50, 115-132.

Happé, F., \& Frith, U. (2006). The weak coherence account: Detail-focused cognitive style in autism spectrum disorders. Journal of Autism and Developmental Disorders, 36, 5-25. 
Jones, W., Carr, K., \& Klin, A. (2008). Absense of preferential looking to the eyes of approaching adults predicts level of social disability in 2-year-old toddlers with autism spectrum disorder. Archives of General Psychiatry, 65, 946-954.

Just, M. A., Keller, T. A., \& Kana, R. K. (2013). A theory of autism based on frontal-posterior underconnectivity. In M. A. Just \& K. A. Pelphrey (Eds.), Development and brain systems in autism (pp. 35-63). New York: Psychology Press.

Just, M. A., Keller, T. A., Malave, V. L., Kana, R. K., \& Varma, S. (2012). Autism as a neural systems disorder: A theory of frontal-posterior underconnectivity. Neuroscience and Biobehavioral Reviews, 36, 1292-1313.

Klin, A., \& Jones, W. (2006). Attributing social and physical meaning to ambiguous visual displays in individuals with higher functioning autism spectrum disorders. Brain \& Cognition, 61, 40-53.

Klin, A., Jones, W. Schultz, R. T., Volkmar, F. R., \& Cohen D. J. (2002a). Defining and quantifying the social phenotype in autism, American Journal of Psychiatry, 159, 895908.

Klin, A., Jones, W., Schultz, R. T., Volkmar, F. R., \& Cohen, D. J. (2002b). Visual fixation patterns during viewing of naturalistic social situations as predictors of social competence in individuals with autism. Archives of General Psychiatry, 59, 809-816.

Kuhn, G. Benson, V., Fletcher-Watson, S., Kovshoff, H., McCormick, C., \& Kirkby, J. (2010) Eye movements affirm: Automatic overt gaze and arrow cueing for typical adults and adults with autism spectrum disorder. Experimental Brain Research, 201, 155-165.

Liversedge, S. P., \& Findlay, J. M. (2000). Saccadic eye movements and cognition. Trends in Cognitive Sciences, 4, 6-14. 
Lord C., Rutter M., DiLavore P. C., Risi S., Gotham K., Bishop S. (2012). Autism diagnostic observation schedule, second edition. Torrance, CA: Western Psychological Services.

Louwerse, A., Tulen, J. H. M., van der Geest, J. N., van der Ende, J., Verhulst, F. C., \& GreavesLord, K. (2014). Autonomic Responses to Social and Nonsocial Pictures in Adolescents With Autism Spectrum Disorder. Autism Research, 7, 17-27. doi:10.1002/aur.1327

Minshew, N. J., \& Goldstein, G. (1998). Autism as a disorder of complex information processing. Mental Retardation and Developmental Disabilities Research Reviews, 4, 129-136.

Minshew, N. J., \& Williams, D. L., (2007). The new neurobiology of autism: cortex, connectivity, and neuronal organization. Archives of Neurology, 64, 945-950.

Minshew, N. J., Goldstein, G., \& Siegel, D. J. (1997). Neuropsychologic funcitoning in autism: Profile of complex information processing disorder. Journal of the International Neuropsychological Society, 3, 303-316.

Pelphrey, K. A., Sasson, N. J., Reznick, J. S., Paul, G., Goldman, B. D., \& Piven, J. (2002). Visual scanning of faces in autism, Journal of Autism and Developmental Disorders, 32, 249-261.

Rayner, K. (2009). Eye movements and attention in reading, scene perception, and visual search. Quarterly Journal of Experimental Psychology, 62, 1457-1506.

Rayner, K., Castelhano, M. S., \& Yang J. (2009). Eye Movements when Looking at Unusual/Weird Scenes: Are there Cultural Differences? Journal of Experimental Psychology: Language, Memory \& Cognition, 35, 254-259. 
Rayner, K., Warren, T., Juhasz, B. J., \& Liversedge, S. P. (2004). The effect of plausibility on eye movements in reading. Journal of Experimental Psychology-Learning Memory and Cognition, 30(6), 1290-1301.

Riby, D. M., \& Hancock, P. J. B. (2009). Do faces capture the attention of individuals with Williams syndrome or Autism? Evidence from tracking eye movements, Journal of Autism and Developmental Disorders, 39, 421-431. doi:10.1007/s10803-008-0641-z

Russell, J. (1997). Autism as an executive disorder. Oxford, UK: Oxford University Press.

Speer, L. L., Cook, A. E., McMahon, W. M., \& Clark, E. (2007). Face processing in children with autism: effects of stimulus contents and type, Autism, 11, 263-277.

Stampe, D. M. (1993). Heuristic filtering and reliable calibration methods for video-based pupiltracking systems. Behavior Research Methods, Instruments, \& Computers. 25, 137142.

The Psychological Corporation, Wechsler Abbreviated Scale of Intelligence (WASI) Manual, (The Psychological Corporation, San Antonio, TX, 1999).

Wolfe, J. M. (1998) What can one million trials tell us about visual search? Psychological Science, 9, 33-39. 
2

3

4

\section{Footnotes}

1. Accuracy errors for the excluded scenes were either a result of a failure to detect a subtle oddity, or were a result of participants thinking the control version of the scenes were odd.

Removing these items from analysis did not change the pattern of effects, but reduced variation in reaction time and eye movement data. 


\section{Tables}

Table 1.

Accuracy and reaction time means (standard deviations).

\begin{tabular}{|c|c|c|c|c|c|c|c|c|}
\hline & \multicolumn{4}{|c|}{ Perceptual } & \multicolumn{4}{|c|}{ Social } \\
\hline & \multicolumn{2}{|c|}{ Normal } & \multicolumn{2}{|c|}{ Weird } & \multicolumn{2}{|c|}{ Normal } & \multicolumn{2}{|c|}{ Weird } \\
\hline & TD & ASD & TD & ASD & TD & ASD & TD & ASD \\
\hline Accuracy & $\begin{array}{c}0.81 \\
(0.20)\end{array}$ & $\begin{array}{c}0.86 \\
(0.10)\end{array}$ & $\begin{array}{c}0.84 \\
(0.20)\end{array}$ & $\begin{array}{c}0.77 \\
(0.17)\end{array}$ & $\begin{array}{c}0.80 \\
(0.17)\end{array}$ & $\begin{array}{c}0.83 \\
(0.12)\end{array}$ & $\begin{array}{c}0.80 \\
(0.21)\end{array}$ & $\begin{array}{c}0.76 \\
(0.24)\end{array}$ \\
\hline $\begin{array}{l}\text { Reaction } \\
\text { Time } \\
(\mathrm{ms})\end{array}$ & $\begin{array}{c}5289 \\
(2372)\end{array}$ & $\begin{array}{c}6024 \\
(2560)\end{array}$ & $\begin{array}{c}3697 \\
(1703)\end{array}$ & $\begin{array}{c}5184 \\
(2228)\end{array}$ & $\begin{array}{c}5117 \\
(2443)\end{array}$ & $\begin{array}{c}6053 \\
(2884)\end{array}$ & $\begin{array}{c}3056 \\
(1270)\end{array}$ & $\begin{array}{c}4633 \\
(2131)\end{array}$ \\
\hline
\end{tabular}




\section{Page 27 of $116 \quad$ Autism Research}

Table 2 .

Eye movement measure means in ms (standard deviations).

\begin{tabular}{|c|c|c|c|c|c|}
\hline & & \multicolumn{2}{|c|}{ Perceptual Oddity } & \multicolumn{2}{|c|}{ Social Oddity } \\
\hline & & $\mathrm{TD}$ & ASD & $\mathrm{TD}$ & ASD \\
\hline Mean Fixation Duration (overall) & & $250(31)$ & 257 (29) & $241(26)$ & $252(33)$ \\
\hline Mean Fixation Count (overall) & & $11(4)$ & $15(6)$ & $9(4)$ & $13(7)$ \\
\hline Elapsed time to target region & ELT & $551(235)$ & $557(144)$ & $600(235)$ & $679(284)$ \\
\hline Fixation count before target region & FCT & $2.32(0.72)$ & $2.49(0.67)$ & $2.62(0.76)$ & $2.68(0.73)$ \\
\hline First fixation duration in target region & FFD & $224(52)$ & $237(42)$ & $232(45)$ & $210(41)$ \\
\hline First gaze duration in target region & FGD & $808(250)$ & $924(437)$ & $630(163)$ & $715(208)$ \\
\hline Mean fixation duration in target region & MFD & $262(43)$ & $274(34)$ & $281(38)$ & $282(45)$ \\
\hline Mean fixation count in target region & MFC & $5.78(2.28)$ & $7.72(2.84)$ & $3.97(1.81)$ & $5.47(2.14)$ \\
\hline Mean number of entries into target region & MNE & $1.93(0.64)$ & $2.32(0.73)$ & $1.62(0.52)$ & $2.07(0.66)$ \\
\hline Mean total time in target region & $\mathrm{TT}$ & $1522(729)$ & $2065(809)$ & $1040(527)$ & $1450(664)$ \\
\hline
\end{tabular}




\section{Figures captions}

Figure 1. Example of social normal (A), social weird (B), perceptual normal (C) and perceptual weird (D) stimuli. The black square represents the target region. Note the black square is not visible during the experiment.

Figure 2. Interaction for first fixation duration (ms) between group and oddity type. Error bars denote + /$1 \mathrm{SE}$. 
RUNNING HEAD: LOOKING, SEEING AND BELIEVING IN AUTISM.

Looking, Seeing and Believing in Autism: Eye Movements reveal how subtle cognitive processing differences impact in the social domain.

Valerie Benson $^{1 *}$, Monica S. Castelhano ${ }^{2}$, Philippa L. Howard ${ }^{1}$, Nida Latif $^{2}$ \& Keith Rayner ${ }^{3}$

${ }^{1}$ AU Psychology, Shackleton Building, Highfield Campus, University of Southampton, UK.

${ }^{2}$ Queen's University, Kingston, ON, Canada

${ }^{3}$ University of California, San Diego, USA

*Correspondence to: vb1@,soton.ac.uk

Telephone: +44 (0) 2380594596 
Lay Abstract

\begin{abstract}
Adults with High Functioning Autism (ASD) viewed scenes with people in them, whilst having their eye movements recorded. The task was to indicate, using a button press, whether the pictures were normal, or in some way weird or odd. Oddities in the pictures were categorized as violations of either perceptual or social norms. Compared to a Typically Developed (TD) control group, the ASD participants were equally able to categorise the scenes as odd or normal, but they took longer to respond. The eye movement patterns showed that the ASD group made more fixations and revisits to the target areas in the odd scenes compared to the TD group. Additionally, when the ASD group first fixated the target areas in the scenes, they failed to initially detect the social oddities. These two findings have clear implications for processing difficulties in ASD for the social domain, where it is important to detect social cues on-line, and where there is little opportunity to go back and recheck possible cues in fast dynamic interactions.
\end{abstract}




\begin{abstract}
Scientific Abstract
Autistic Spectrum Disorder (ASD) and Typically Developed (TD) adult participants had to decide whether scenes were 'odd', 'unusual' or 'weird'. All scenes contained people, with 'oddness' operationally defined as either perceptual or social. There were no group differences in accuracy, but the ASD group were slower overall to respond manually. Eye movements revealed that the ASD group made more fixations overall and more re-fixations into the target regions of the pictures, for both conditions. Importantly, as indexed by the first fixation duration the ASD group failed to 'pick up' immediately on what was 'odd' for the social violations. The propensity to 'go back' and re-fixate targets, coupled with a failure to initially detect social violations, has obvious significance for ASD in fast dynamic social communication.
\end{abstract}

Key phrases: Autism Spectrum Disorder, Eye movements, On-line cognitive processing, Social and Perceptual Oddities. 


\section{Autism Research}

Looking, Seeing and Believing in Autism: Eye Movements reveal how subtle cognitive processing differences impact in the social domain.

Autism spectrum disorders (ASD) are lifelong neuro-developmental conditions characterized by repetitive behaviours and impairments in interaction and communication (American Psychiatric Association, 2013). Incidence rates are rising across the globe with the prevalence rate in the UK approximately 10 per 1000 (1\%) individuals (Brugha et al., 2011) and the UK National Audit office recently called for more research into adults with ASD (Clarke et al., 2009).

High functioning adults with ASD are similar to typically developed (TD) adults in terms of performance on pencil and paper and IQ tests, however in the social domain evidence suggests an inability to process social information in a typical fashion. Eye movement studies report unusual gaze behaviour for faces appearing on their own (Dalton et al., 2005), in social scenes (Riby \& Hancock, 2009) or in dynamic movie clips (Klin \& Jones, 2006; Speer et al., 2007). Research consistently reports a lack of spontaneous gaze fixation towards the eyes (Jones et al., 2008; Klin et al., 2002a; Pelphrey et al., 2002). However, where one study reports impairments, say for face processing (Klin et al., 2002b), another study does not (Fletcher-Watson et al., 2009). Moreover, lack of attentional modulation for social stimuli (Bird et al., 2006) may be overridden, as when cued, attention may be allocated to faces in a more 'typical' manner (BarHaim et al., 2006).

A recent scene perception study (Louwerse et al., 2014) reported that abnormal autonomic responses in ASD are unlikely to underpin social processing impairments. It is interesting then to ask whether impairments in the cognitive domain in ASD (Happé \& Frith, 2006; Minshew et al., 1997; Russell, 1997) could account for impairments in the social domain? 
Current cognitive theories of ASD include, among others, Weak Central Coherence (WCC) theory (Frith \& Happé, 1994; Happé \& Frith, 2006) which proposes that a reduced sensitivity to global context in favour of focussing on details, results in integration difficulties in ASD. Executive function theory (Russell, 1997), proposes that ASD are impaired at switching their focus of attention, and may engage is perseverative behaviour. Alternatively, the Complex Information Processing theory (Minshew \& Goldstein, 1998) proposes that individuals with ASD have impairments in processing complex information with intact processing for tasks that require simple (rule based) processing. Finally, the Extreme Male Brain (EMB) theory (Baron-Cohen, 2002) posits that individuals with ASD have above average levels of systemizing, that contribute to the non-social characteristics of the disorder, coupled with below average levels of empathizing, which results in atypical social functioning. These contemporary theories of ASD can, for different reasons, offer some account offor ineffective social functioning in ASD. However, , but sparse research to date has-very few investigations have examineded time course processing differences as these occur on-line in $\mathrm{ASD}_{-}$, and how these might impact in the social domain.

Our studies investigating cognitive processing in adults with ASD (Au-Yeung et al., 2011; Au-Yeung et al., 2013; Benson et al., 2012; Benson et al., 2009; Benson \& FletcherWatson, 2011) have found subtle on-line processing differences, as revealed by eye movements, for a range of tasks that are more in line with Minshew and Goldsteins (1998) theory, than with any other. For example, there are more similarities than differences in eye movements between TD and ASD groups for simple rule based tasks in the attentional (Kuhn et al., 2010) and the social domains (Benson \& Fletcher-Watson, 2011). Whereas for more complex abstract reasoning tasks where there is ambiguity with relation to target items (Au-Yeung et al., 2013), or 


\section{Autism Research}

where value judgements rely on top down knowledge (Benson et al., 2012), there are early processing differences indicating that initial orienting to, and immediate detection of weird target items in scenes is absent in ASD. Moreover, the time to respond and to locate the weird target items was modulated by whether or not the pictures had people in them (Benson et al., 2012). These differences have potential forto impact onin everyday activities, since the time course for detection of socially relevant information is crucial for successful everyday interactions.

The aim of the present study was to examine whether increased times to find and initially detect an unusual target in a scene would be modulated by whether the violation that rendered the target unusual or odd could be categorised as social or perceptual. We employed a similar paradigm to that used in a previously reported study (Rayner et al., 2009) where single scenes, all with people in them, were presented in isolation and the task was to indicate whether each picture was normal or in some way weird unusual or odd.

Insert Figure 1. about here

Social oddities in the pictures were operationally defined as violations of social norms e.g. someone in the picture throwing a baby in the air. Access to top down knowledge is required in that condition to inform a subjective value judgement as to whether a social violation is present in the picture. No value judgement as to whether a violation infringes some implicit or explicit social rule is needed for the perceptual oddities in this study, which are operationally defined as physical violations e.g. someone in the picture has a limb positioned back to front. See supplementary information for Materials-and Methods. Figure 1 shows an example of the stimuli.

\section{Method}




\section{Participants}

ASD participants ( $n=24$ age $18-52$ years, 20 males), with a formal diagnosis of either Autistic Disorder ( $n=3)$ or Asperger's Syndrome were recruited from local charitable organisations. TD participants ( $\mathrm{n}=24$, age $20-52$ years, 20 males) were volunteers from the local community. All participants were paid and had normal or corrected to normal vision. Each ASD participant completed module 4 of the Autism Diagnostic Observation Scale 2 (Lord et al., 2012) and five (3 males and 2 females with a diagnosis of Asperger's Syndrome) failed to meet the Autism Spectrum criteria. To ensure maximum homogeneity in our study those five participants were excluded from the analyses, resulting in a final sample of 19 ASD participants. Both groups completed the Autism-spectrum Quotient (AQ; Baron-Cohen et al., 2001) and the ASD participants reported a higher number of autistic traits $t(41)=8.04, p<.001$ (TD $M=$ $15.42, S D=7.55$; ASD $M=35.32, S D=8.45)$. The two groups did not differ in age $t(41)=$ $0.31, p=.759(\mathrm{TD} M=30.29, S D=10.20 ;$ ASD $M=31.26, S D=10.29)$, performance IQ $t(41)$ $=0.21, p=.832(\operatorname{TD} M=111.79, S D=12.01 ;$ ASD $M=110.95, S D=13.84)$, verbal IQ $t(41)=$ $1.21, p=.234(\mathrm{TD} M=116.96, S D=10.65$, ASD $M=112.26, S D=14.84)$, or full scale IQ $t$ $(46)=0.87, p=.392(\operatorname{TD} M=116.04, S D=9.98, \operatorname{ASD} M=112.95, S D=13.48)$, as measured by two performance (Block Design and Matrix Reasoning) and verbal components (Vocabulary and Similarity) of the Weschler Abbrevated Scale of Intelligence (The Psychological Corporation, 1999).

\section{Eye movement recording}

Eye movements were sampled at $1000 \mathrm{~Hz}$ using an Eyelink tracker with spatial accuracy

of less than $1 / 4^{\text {th }}$ degree (S.R. Research Ltd, Canada). The start and end of saccades were 


\section{Materials}

In total, 96 full-colour photographs were used as stimuli, all of which contained people. In both the perceptual and social oddity conditions there were 48 pictures (24 weird and 24 normal). Perceptual oddities in the pictures violated a physical norm e.g. someone in the picture has a limb positioned back to front, or a vehicle was in an unexpected position e.g midway through the upper wall of a building. Social oddities in the pictures violated a social norm e.g. someone in the picture throwing a baby in the air, or someone with a cabbage on a lead. A small number of the pictures were from a previous study (Benson et al., 2012) and the remainder were collected from the internet and included either a perceptual or social oddity. Adobe Photoshop was used to prepare a normal version of each scene in which the oddity was replaced with either the background elements or with another suitable object. The scenes typically had a focal object or a central group of objects. Figure 1 shows an example of a normal and a socially odd scene, 
and below those examples, a normal and a perceptually odd scene. The target regions for these scenes are outlined in black - but these black outlines were not visible during the presentation of the scenes in the experiment.

A norming study was conducted to verify the validity of the images used to examine the processing of social and perceptual violations. Twenty undergraduates from Queen's university participated ( $M$ age: 19.8years, $S D: 1.71,10$ Females) with five participants in each of the four conditions (counterbalanced conditions: weird/normal and right/left button response). Stimuli were displayed on a 21 inch CRT monitor with a refresh rate of $100 \mathrm{~Hz}$ and the experiment was run using E-Prime software. Participants were presented with a fixation cross for $500 \mathrm{~ms}$ followed by the presentation of the image for 20 seconds or until a keyboard " $\mathrm{Y}$ ' (yes) or "N" (N) was made. Participants had to indicate as quickly as possible whether the presented image was "odd", "weird" or "unusual" in some way. Results-The proportion of identified images indicated that on average, the social normal $(M=0.86, S D=0.15)$ and social weird $(M=0.84$, $S D=0.17)$, perceptual normal $(M=0.82, S D=0.17)$ and perceptual weird $(M=0.77, S D=0.19)$ images were identified correctly.

\section{Design}

For the behavioural measures of Accuracy and RT a mixed design with Picture Type (weird versus normal) and Oddity Type (social versus perceptual) as within participants variables, and Group (ASD versus TD) as a between participants variable was employed for the experiment. On condition that accuracy was equivalent for both normal and weird pictures, for the eye movement measures the main focus of interest was to compare social versus perceptual oddities in both groups. Therefore a mixed design with Oddity Type (social versus perceptual) as 
a within participants variable, and Group (ASD versus TD) as a between participants variable was employed in the eye movement analysis.

\section{Procedure}

All participants were shown a hard copy of an example picture from each condition prior to completing the task, to ensure comprehension of the task and the ability to respond appropriately. The specific instruction was 'for each of the following pictures, please indicate whether you think the picture is odd, unusual or strange in some way'.

For the task, 48 pictures were presented randomly to either the left or the right of the centre of a 19inch colour monitor, at a distance of $65 \mathrm{~cm}$ for $20 \mathrm{~s}$ or until a button press response was made. Prior to the onset of a picture, participants fixated a cross that was presented on the opposite side of the screen to where the picture was set to appear, to ensure all participants started at the same point in each trial. Once participants fixated this cross, the experimenter initiated each trial. Picture type (normal vs. weird) and, the position of the picture (left or right on the display) were counterbalanced across items. The buttons used to respond either yes or no (left or right trigger button) was counterbalanced across participants.

\section{Data preparation and analyses}

For each 'odd' picture we created an interest region around the target item and the corresponding region of the same size in the counterpart 'normal' picture. Regions of interest were created using the approximate average regions from those identified and manually selected by participants who took part in the norming experiment. 
Eyelink software was used to detect saccades and fixations using a default velocity criterion and in-house software was used to calculate the distribution of fixations falling into the target region. Five items were removed from analysis because for these scenes, participant accuracy scores were significantly below chance ${ }^{1}$. In addition, each participant was asked whether they had seen any of the scenes previously, and if so these scenes were also removed. This resulted in a loss of $2.3 \%$ of trials. Fixations below $90 \mathrm{~ms}$ and above $1200 \mathrm{~ms}$ were classed as outliers and removed; resulting in a loss of $5.13 \%$ of the fixation data.

\section{Results}

For the principal analyses we compared manual reaction times and accuracy (see Table 1), as well as a number of eye movement measures, such as, the time taken to begin fixating in the target region (ELT), the duration of the first fixation when the eyes landed in the target region (FFD) and the mean time (MFD) spent fixating in the target area (see Table. 2). Global eye movement measures for the target region, such as mean fixation duration and mean number of fixations over all trials were also analysed, Finally, baseline measures (not just target related), were calculated across the duration of the trials, to check for overall sampling differences between the ASD and TD participants (see Table 2). See supplementary information for methods and materials.

\section{Behavioural Data}

Accuracy. There was no effect of oddity type (perceptual vs. social) $F(1,41)=1.44, p=$ $.237, \eta_{\mathrm{p}}^{2}=.03$, picture type (normal or weird) $F(1,41)=0.94 p=.339, \eta_{\mathrm{p}}^{2}=.02$, or group, $F(1$, $41)=.06, p=.808, \eta_{\mathrm{p}}^{2}<.01$ on accuracy rates, and no interactions $\left(F_{\mathbf{S}}<2.39, p^{\prime} s>.130\right)$. At 


\section{Autism Research}

least in terms of accuracy, performance was the same for both groups. For means and standard deviations for behavioural data see Table 1 .

Response Time (RT). A main effect of oddity type $F(1,41)=8.16, p=.007, \eta_{\mathrm{p}}{ }^{2}=.17$, showed participants took longer to respond when presented with a perceptual oddity in comparison to a social oddity. Longer response times for perceptual oddities could reflect that these 'impossible', physical violations were so unusual that they resulted in more time spent looking at them prior to responding. See below for confirmation of that in the eye movement data. There was also a main effect of picture type $F(1,41)=24.03, p<.001, \eta_{\mathrm{p}}{ }^{2}=.37$, with participants taking longer to respond when the picture was normal in comparison to when it was weird, in line with longer reaction times for target absent trials in standard visual search tasks (Wolfe, 1998) A significant oddity type $\mathrm{x}$ picture type interaction $F(1,41)=4.22, p=.046, \eta_{\mathrm{p}}{ }^{2}$ $=.09$, demonstrated that the difference between RTs for perceptual and social pictures was reliable for odd (TD; $t$ (23) 2.62, $p=.015$, ASD; $t(18)=2.98, p=.008$ ) but not for normal pictures (TD; $t(23) 0.94, p=.355$, ASD: $t(18)=0.08, p=.935)$. A main effect of group $F(1$, 41) $=4.07, p=.050, \eta_{\mathrm{p}}{ }^{2}=.09$, showed the ASD participants had longer reaction times, in line with a previous study (Benson et al., 2012 25). There were no significant group interactions $(F \mathrm{~s}$ vs social oddities. See Table 1 for the means. The eye movement data below reveals subtle processing differences that contribute to this overall slowed manual responding in the ASD group.

\section{Eye Movement Data}


Having shown that all participants could distinguish between 'odd' and 'normal' pictures, normal picture trials were excluded from the eye movement analyses resulting in a 2 (group: ASD versus TD) X 2 (oddity type: social versus perceptual) design to investigate on-line processing differences between the two groups for the two types of oddities.

Baseline eye movement measures, calculated across the full length of trials indicated no group difference in mean fixation duration $F(1,41)=1.14, p=.293, \eta_{\mathrm{p}}{ }^{2}=.03$, but the ASD group made more fixations overall $F(1,41)=9.44, p=.004, \eta_{\mathrm{p}}{ }^{2}=.19$, corresponding to the increased RT's reported above. Table 2 presents a summary of all the eye movement measures.

Elapsed time to the target region (ELT). A marginal effect of oddity type $F(1,41)=3.99, p=$ $.053, \eta_{\mathrm{p}}{ }^{2}=.09$, on the time taken from the onset of each trial display to the start of the first fixation on the target region, revealed both groups took less time to fixate the target region when the oddity was perceptual in comparison to social in nature. The impossible nature of the perceptual violation may have increased the salience of the target region but $\underline{T}$ there was no effect of group $F(1,41)=0.59, p=.446, \eta_{\mathrm{p}}{ }^{2}=.01$, and no interaction $F(1,41)=0.72, p=.401, \eta_{\mathrm{p}}{ }^{2}=$ .02 .

First Fixation Duration (FFD) on target region. There was no effect of oddity type $F(1,41)=$ $1.84, p=.182, \eta_{\mathrm{p}}^{2}=.04$ or group $F(1,41)=0.15, p=.699, \eta_{\mathrm{p}}^{2}<.01$ but a significant interaction $F(1,41)=6.72, p=.013, \eta_{\mathrm{p}}{ }^{2}=.14$ (See Figure 2) revealed that FFDs were equivalent for perceptual and social oddities for TD participants $t(23)=0.86, p=.399, d=.16$, but for ASD participants FFDs for social oddities were significantly shorter, in comparison to perceptual oddities, $t(18)=2.99, p=.008, d=.65$, see Figure 2 . Given that the FFD is a measure of early 
processing ( (Liversedge \& Findlay, 2000; Rayner, 1998; Rayner, 2009).31 33), this finding indicates that the ASD group did not detect the social oddities as quickly as the perceptual oddities on initial fixation, whereas TD participants detected both types of oddity equally fast.

Insert Figure 2. about here

First gaze duration (FGD) in the target region. The FGD is the elapsed time from when the target region is first fixated until the eye moves on to a new location. FGD includes multiple fixations in the target region reflects an overt strategy to re-examine an area. A main effect of oddity type, $F(1,41)=12.10, p=.001, \eta_{\mathrm{p}}{ }^{2}=.23$ demonstrated that both groups spent more time inspecting perceptual oddities, again highlighting the salience of the perceptual oddities in comparison to social oddities in this study. However, there was no group effect $F(1,41)=2.43$, $p=.127, \eta_{\mathrm{p}}^{2}=.06$, or interaction $F(1,41)=.08, p=.785, \eta_{\mathrm{p}}^{2}<.01$.

Mean Fixation Count in the target region. If the ASD are slower to respond manually, but do not take longer to begin inspecting the target region, then extra time to respond might reflect more fixations in the target region. A main effect of oddity type $F(1,41)=64.27, p<.001, \eta_{\mathrm{p}}{ }^{2}=$ .61 , demonstrated more fixations in the target region when the oddity was perceptual, rather than social. A main effect of group $F(1,41)=6.99, p=.012, \eta_{\mathrm{p}}{ }^{2}=.15$ showed that the ASD participants-group made more fixations than the TD group in the target regions, for both types of oddities-overall. However no interaction was observed $F(1,41)=0.77, p=.386, \eta_{\mathrm{p}}{ }^{2}=.02$ indicating that the ASD group made more fixations in the target regions for both types of oddities.

Formatted: Highlight
Formatted: Font: (Default) Times New
Roman, 12 pt
Formatted: Font: (Default) Times New
Roman, 12 pt, Not Italic
Formatted: Font: Not Italic
Formatted: Font: (Default) Times New
Roman, 12 pt
Formatted: Highlight


Mean Fixation Duration in the target region. Longer mean fixation durations indicate that more processing has been done during the task. A main effect of oddity type $F(1,41)=8.22, p=$ $.007, \eta_{\mathrm{p}}{ }^{2}=.17$ showed significantly longer fixations for social, compared to perceptual oddity target regions. This suggests that processing social oddities was more effortful, and since there was no main effect of group $F(1,41)=0.34, p=.562, \eta_{\mathrm{p}}{ }^{2}<.01$, and no interaction $F(1,41)=$ $1.12, p=.297, \eta_{\mathrm{p}}{ }^{2}=.03$, both groups on average fixated the social oddity target region for longer than the perceptual target region. Thus, while the perceptual oddities attracted more fixations overall, individual fixations were longer for the social target region.

This interesting juxtaposition, although unrelated to the main aim of this study, suggests that difficulty associated with processing each oddity type manifests in different eye movement patterns: one reflecting an overt strategy (fixation count) to re-inspect the target area, and one reflecting more in depth on-line cognitive processing (fixation duration) on the target areas.

Mean Number of Entries to the target region. Since there were no group differences in FGD, and no increased MFD, both reported above, the delayed RT in ASD might reflect repeated visits to inspect the target region?

A main effect of oddity type was found $F(1,41)=9.83, p=.003, \eta_{\mathrm{p}}{ }^{2}=.19$, with the target region entered more often for perceptual oddities in comparison to social oddities. In addition, a main effect of group was found $F(1,41)=5.72, p=.021, \eta_{\mathrm{p}}{ }^{2}=.12$, with ASD participants entering the target regions significantly more than the TD group, before making a response. There was no interaction $F(1,41)=0.13, p=.717, \eta_{\mathrm{p}}^{2}<.01$. 
Mean Total Time in the target region. A higher mean total time means that more attention to, and processing of, information in that region was carried out overall. This measure should complement the measures reported above - namely, that if there are more fixations overall in ASD then the Total Time spent fixating in the target region should be longer in ASD.

A main effect of oddity type $F(1,41)=34.89, p<.001, \eta_{\mathrm{p}}{ }^{2}=.46$, showed perceptual oddities received more overall processing time, in line with the previous measures for the perceptual oddities. In addition, a main effect of group $F(1,41)=6.41, p=.015, \eta_{\mathrm{p}}{ }^{2}=.14$ revealed that the ASD participants spent a longer period of time inspecting the target regions for both perceptual and social oddities. There was no interaction $F(1,41)=0.51, p=.481, \eta_{\mathrm{p}}{ }^{2}=.01$.

As above, this finding reflects a greater number of entries to the target region overall by the ASD group prior to making their decision as to whether the picture under inspection was weird, odd or unusual, irrespective of the nature of the violation (perceptual or social).

\section{Discussion}

A principal aim of the study was to investigate whether increased times in ASD to find and initially detect an unusual target in a scene containing people would be modulated by whether the violation could be categorised as social or perceptual.

Accuracy rates were equivalent and high for both groups, but the ASD group took longer to respond manually. It was hypothesised that the eye movement data would reveal the cause of this delayed response in ASD, since eye movements reflect how information is processed on-line (Liversedge \& Findlay, 2000; Rayner, 1998; 2009). The data showed that our ASD group did not take longer to find the target regions, but that they scanned the scenes more prior to responding manually, as shown by an increased number of fixations throughout each scene presentation. 
Furthermore, these increased fixations were not random, but were made to the target regions of both social and perceptually odd pictures. Thus, increased fixations reflect a propensity to reinspect the target regions prior to making a response in ASD, regardless as to the type of oddity in the scenes. It should be noted here that the oddity detection task in the current study provides the ASD participants with structure that is not typically available in real-life. As such, the task is not implicit and the knowledge that oddities might be present could also be a factor that drives the repeated scanning in ASD.

The eye movement patterns also showed that our ASD group failed to immediately detect social oddities, thus looking at but not seeing them. An obvious question from this finding is whether the failure to quickly spot subtle social oddities in ASD reflects an inability to react or respond appropriately to social cues in everyday communication? This one impairment in itself could potentially account for the significant problems experienced in dynamic social interactions in this high functioning population. For example, a failure to immediately spot social cues would result in a failure to react or respond to such cues thus resulting in an inability to both keep track of what is going on in social interactions, and a failure to engage appropriately or effectively with other people:-

In relation to current theories that underpin ASD our findings do not fit with WCC theory

(Frith \& Happé, 1994; Happé \& Frith. 2006), since clearly context can be taken into account in ASD during task completion. Moreover, the slowness to respond in ASD is not reflected in longer times to find the target, and the increased fixations in ASD were seen for both perceptuat and social oddities in the scenes. It may be that repeated visits to the target area indicate integration problems in ASD, but even if that were the case, such problems are not restricted to social processing per se. Neither do the support the executive function theory (Russell, 1997), as
Formatted: Font: (Default) Times New Roman, 12 pt

Formatted: Font: (Default) Times New Roman, 12 pt

Formatted: Font: (Default) Times New Roman, 12 pt 


\section{Autism Research}

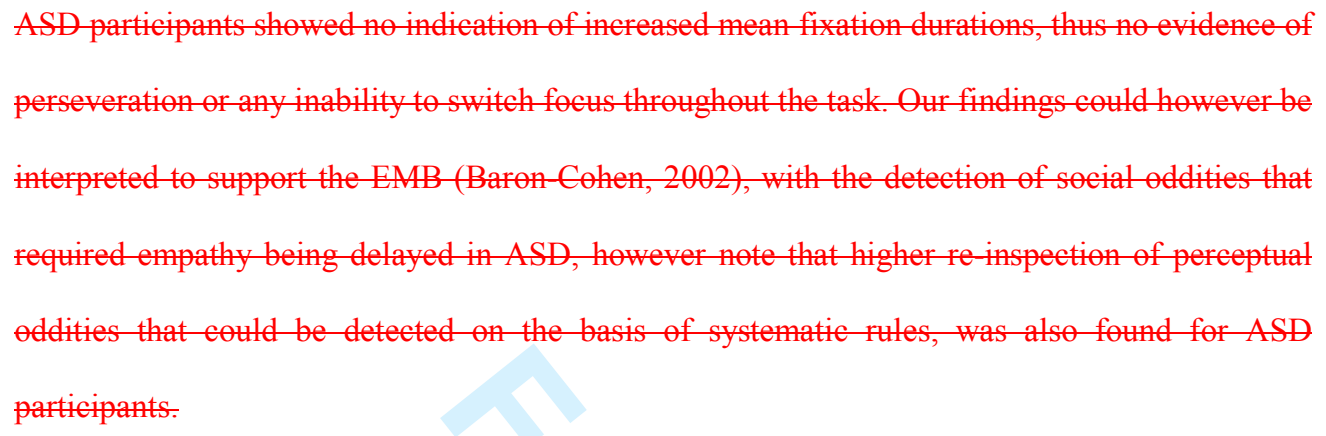

If social oddities are more complex to detect in ASD, then the Complex Information Processing theory (Minshew \& Goldstein, 1998) might explain the failure to detect the social oddity on first fixation. This lack of immediate detection could reflect the less obvious and more 'inferential' nature of the social oddities, which required more complex reasoning, at least in the

ASD group, for detection. It is important to note that social rules are often implicit, sometimes ambiguous, and cannot always be understood by following a script or flow chart as in the case for logical rule based tasks. At a neurobiological level, it has been postulated that that impairments in cognitive processing in ASD reflect slow information transmission rates for high level cognitive tasks, resulting from underconnectivity between frontal-posterior cortical regions (Just et al., 2013; Just et al., 2012; Minshew \& Williams, 2007). Since underconnectivity reduces the rate of inter-region information transfer, it could be that in social communication tasks that rely on the fast detection of implicit, often ambiguous cues, a failure to be able to transmit information across different cortical areas quickly, might mean that these cues are missed, rendering inappropriate responses in ASD.

The findings of repeated scanning of the target areas, coupled with an inability to immediately recognise social violations upon initial fixation, reflect how eye movements can reveal subtle processing differences in ASD. In everyday social communication in the real world
Formatted: Not Highlight

Formatted: Font: (Default) Times New Roman, 12 pt, Not Highlight

Formatted: Not Highlight 
there is no opportunity to 'go back' to check if one has missed an important cue. Social processing in such circumstances must be carried out on-line, and our eye movement data clearly show that ASD are unable to do this quickly. The strategy of re-checking their interpretation, and thus not believing what they have seen, before responding, may be related to cortical underconnectivity in ASD. In everyday terms, these findings have obvious consequences for fast dynamic social interactions, and offer an account as to why high functioning adults with ASD experience difficulty in the social domain. 


\section{Acknowledgments}

This work was supported by a grant awarded to the second author from the Natural Sciences and Engineering Research Council of Canada, the Canadian Foundation for Innovation, the Ontario Ministry of Research and Innovation, and Queen's University Senate Advisory Research Committee, and an Experimental Psychology Society study visit grant awarded to the third author. 
Au-Yeung, S. K., Kaakinen, J. K., Benson, V. (2013). Cognitive perspective taking during scene perception in autism spectrum disorder: evidence from eye movements. Autism Research, 7, 84-93.

Bar-Haim, Y., Shulman, C., Lamy, D., \& Reuveni, A. (2006). Attention to eyes and mouth in high-functioning children with autism, Journal of Autism and Developmental Disorders, $36,131-137$.

Baron-Cohen, S. (2002). The extreme male brain theory of autism. Trends in Cognitive Science, $6,248-254$.

Baron-Cohen, S., Wheelwright, S., Skinner, R., Martin, J., \& Clubley, E. (2001). The AutismSpectrum Quotient (AQ): Evidence from Asperger syndrome/high-functioning autism, males and females, scientists and mathematicians. Journal of Autism and Developmental Disorders, 31(1), 5-17.

Benson, V., \& Fletcher-Watson, S. (2012). Eye Movements in Autism. In: Liversedge, S. P., Gilchrist, I. D., Everling, S., editors. The Oxford Handbook of Eye Movements, Oxford UK: Oxford University Press, chap 39.

Benson, V., Castelhano, M. S., Au-Yeung, S. K., \& Rayner K. (2012). Eye movements reveal no immediate "WOW" ("which one's weird") effect in autism spectrum disorder, Quarterly 
Journal of Experimental Psychology, 65, 1139-1150.

Benson, V., Piper, J., \& Fletcher-Watson, S. (2009). Atypical saccadic sampling in autistic spectrum disorder, Neuropsychologia, 47, 1178-1182, (2009).

Bird, G., Catmur, C., Silani, G., Frith, C., \& Frith, U. (2006). Attention does not modulate neural responses to social stimuli in autism spectrum disorders. Neuroimaging, 15, 1614-1624.

Brugha, T. S., McManus, S., Bankart, J., Scott, F., Purdon S., et al. (2011). Epidemiology of autism spectrum disorders in adults in the community in England. Archives of Genereal Psychiatry, 68, 459-65.

Clark, F., Scharaschkin, A., Xu, D. (2009). Supporting autism through adulthood, National Audit Office.

Dalton, K. M., Nacewicz, B. M., Johnstone, T., Schaefer, H. S., Gernsbacher, M. A., Goldsmith, H. H., Alexander, A. L., \& Davidson, R. J. (2005). Gaze fixation and the neural circuitry of face processing in autism. Nature Neuroscience, 8, 519-526.

Fletcher-Watson, S., Leekam, S. R., Benson, V., Frank, M. C., \& Findlay, J. M. (2009). Eyemovements reveal attention to social information in autism spectrum disorder. Neuropsychologia, 47, 248-257.

Fletcher-Watson., Leekam, S.R., Benson, V., Frank, M.C., \& Findlay, J.M. (2009). Eyemovements reveal attention to social information in autism spectrum disorder. Neuropsychologia, 47, 248-257.

Frith, U., \& Happé, F. (1994). Autism; Beyond the theory of mind. Cognition, 50, 115-132. Happé, F., \& Frith, U. (2006). The weak coherence account: Detail-focused cognitive style in autism spectrum disorders. Journal of Autism and Developmental Disorders, 36, 5-25. 
Jones, W., Carr, K., \& Klin, A. (2008). Absense of preferential looking to the eyes of approaching adults predicts level of social disability in 2-year-old toddlers with autism spectrum disorder. Archives of General Psychiatry, 65, 946-954.

Just, M. A., Keller, T. A., \& Kana, R. K. (2013). A theory of autism based on frontal-posterior underconnectivity. In M. A. Just \& K. A. Pelphrey (Eds.), Development and brain systems in autism (pp. 35-63). New York: Psychology Press.

Just, M. A., Keller, T. A., Malave, V. L., Kana, R. K., \& Varma, S. (2012). Autism as a neural systems disorder: A theory of frontal-posterior underconnectivity. Neuroscience and Biobehavioral Reviews, 36, 1292-1313.

Klin, A., \& Jones, W. (2006). Attributing social and physical meaning to ambiguous visual displays in individuals with higher functioning autism spectrum disorders. Brain \& Cognition, 61, 40-53.

Klin, A., Jones, W. Schultz, R. T., Volkmar, F. R., \& Cohen D. J. (2002a). Defining and quantifying the social phenotype in autism, American Journal of Psychiatry, 159, 895908.

Klin, A., Jones, W., Schultz, R. T., Volkmar, F. R., \& Cohen, D. J. (2002b). Visual fixation patterns during viewing of naturalistic social situations as predictors of social competence in individuals with autism. Archives of General Psychiatry, 59, 809-816.

Kuhn, G. Benson, V., Fletcher-Watson, S., Kovshoff, H., McCormick, C., \& Kirkby, J. (2010) Eye movements affirm: Automatic overt gaze and arrow cueing for typical adults and adults with autism spectrum disorder. Experimental Brain Research, 201, 155-165.

Liversedge, S. P., \& Findlay, J. M. (2000). Saccadic eye movements and cognition. Trends in Cognitive Sciences, 4, 6-14. 
Lord C., Rutter M., DiLavore P. C., Risi S., Gotham K., Bishop S. (2012). Autism diagnostic observation schedule, second edition. Torrance, CA: Western Psychological Services.

Louwerse, A., Tulen, J. H. M., van der Geest, J. N., van der Ende, J., Verhulst, F. C., \& GreavesLord, K. (2014). Autonomic Responses to Social and Nonsocial Pictures in Adolescents With Autism Spectrum Disorder. Autism Research, 7, 17-27. doi:10.1002/aur.1327

Minshew, N. J., \& Goldstein, G. (1998). Autism as a disorder of complex information processing. Mental Retardation and Developmental Disabilities Research Reviews, 4, $129-136$.

Minshew, N. J., \& Williams, D. L., (2007). The new neurobiology of autism: cortex, connectivity, and neuronal organization. Archives of Neurology, 64, 945-950.

Minshew, N. J., Goldstein, G., \& Siegel, D. J. (1997). Neuropsychologic funcitoning in autism: Profile of complex information processing disorder. Journal of the International Neuropsychological Society, 3, 303-316.

Pelphrey, K. A., Sasson, N. J., Reznick, J. S., Paul, G., Goldman, B. D., \& Piven, J. (2002).

Visual scanning of faces in autism, Journal of Autism and Developmental Disorders, 32, $249-261$.

Rayner, K. (2009). Eye movements and attention in reading, scene perception, and visual search. Quarterly Journal of Experimental Psychology, 62, 1457-1506.

Rayner, K., Castelhano, M. S., \& Yang J. (2009). Eye Movements when Looking at Unusual/Weird Scenes: Are there Cultural Differences? Journal of Experimental Psychology: Language, Memory \& Cognition, 35, 254-259. 
Rayner, K., Warren, T., Juhasz, B. J., \& Liversedge, S. P. (2004). The effect of plausibility on eye movements in reading. Journal of Experimental Psychology-Learning Memory and Cognition, 30(6), 1290-1301.

Riby, D. M., \& Hancock, P. J. B. (2009). Do faces capture the attention of individuals with Williams syndrome or Autism? Evidence from tracking eye movements, Journal of Autism and Developmental Disorders, 39, 421-431. doi:10.1007/s10803-008-0641-z

Russell, J. (1997). Autism as an executive disorder. Oxford, UK: Oxford University Press.

Speer, L. L., Cook, A. E., McMahon, W. M., \& Clark, E. (2007). Face processing in children with autism: effects of stimulus contents and type, Autism, 11, 263-277.

Stampe, D. M. (1993). Heuristic filtering and reliable calibration methods for video-based pupil- tracking systems. Behavior Research Methods, Instruments, \& Computers. 25, 137142.

The Psychological Corporation, Wechsler Abbreviated Scale of Intelligence (WASI) Manual, (The Psychological Corporation, San Antonio, TX, 1999).

Wolfe, J. M. (1998) What can one million trials tell us about visual search? Psychological Science, 9, 33-39.
Formatted: Font: (Default) Times New Roman, 12 pt, Not Italic

Formatted: Indent: Left: 0", Hanging: 0.5", Line spacing: Double

Formatted: Font: (Default) Times New Roman, 12 pt

Formatted: Font: (Default) Times New Roman, 12 pt, Not Italic 


\section{Footnotes}

1. Accuracy errors for the excluded scenes were either a result of a failure to detect a subtle oddity, or were a result of participants thinking the control version of the scenes were odd.

Removing these items from analysis did not change the pattern of effects, but reduced variation in reaction time and eye movement data. 
Tables

Table 1.

Accuracy and reaction time means (standard deviations).

\begin{tabular}{|c|c|c|c|c|c|c|c|c|}
\hline & \multicolumn{4}{|c|}{ Perceptual } & \multicolumn{4}{|c|}{ Social } \\
\hline & \multicolumn{2}{|c|}{ Normal } & \multicolumn{2}{|c|}{ Weird } & \multicolumn{2}{|c|}{ Normal } & \multicolumn{2}{|c|}{ Weird } \\
\hline & TD & ASD & $\mathrm{TD}$ & ASD & TD & ASD & TD & ASD \\
\hline Accuracy & $\begin{array}{c}0.81 \\
(0.20)\end{array}$ & $\begin{array}{c}0.86 \\
(0.10)\end{array}$ & $\begin{array}{c}0.84 \\
(0.20)\end{array}$ & $\begin{array}{c}0.77 \\
(0.17)\end{array}$ & $\begin{array}{c}0.80 \\
(0.17)\end{array}$ & $\begin{array}{c}0.83 \\
(0.12)\end{array}$ & $\begin{array}{c}0.80 \\
(0.21)\end{array}$ & $\begin{array}{c}0.76 \\
(0.24)\end{array}$ \\
\hline $\begin{array}{l}\text { Reaction } \\
\text { Time } \\
(\mathrm{ms})\end{array}$ & $\begin{array}{c}5289 \\
(2372)\end{array}$ & $\begin{array}{c}6024 \\
(2560)\end{array}$ & $\begin{array}{c}3697 \\
(1703)\end{array}$ & $\begin{array}{c}5184 \\
(2228)\end{array}$ & $\begin{array}{c}5117 \\
(2443)\end{array}$ & $\begin{array}{c}6053 \\
(2884)\end{array}$ & $\begin{array}{c}3056 \\
(1270)\end{array}$ & $\begin{array}{c}4633 \\
(2131)\end{array}$ \\
\hline
\end{tabular}


Table 2 .

Eye movement measure means in ms (standard deviations).

\begin{tabular}{llccccc}
\hline & & \multicolumn{2}{c}{ Perceptual Oddity } & & \multicolumn{2}{c}{ Social Oddity } \\
\cline { 3 - 5 } \cline { 5 - 6 } & & TD & ASD & & TD & ASD \\
\hline Mean Fixation Duration (overall) & & $250(31)$ & $257(29)$ & & $241(26)$ & $252(33)$ \\
Mean Fixation Count (overall) & & $11(4)$ & $15(6)$ & & $9(4)$ & $13(7)$ \\
Elapsed time to target region & ELT & $551(235)$ & $557(144)$ & & $600(235)$ & $679(284)$ \\
Fixation count before target region & FCT & $2.32(0.72)$ & $2.49(0.67)$ & & $2.62(0.76)$ & $2.68(0.73)$ \\
First fixation duration in target region & FFD & $224(52)$ & $237(42)$ & & $232(45)$ & $210(41)$ \\
First gaze duration in target region & FGD & $808(250)$ & $924(437)$ & & $630(163)$ & $715(208)$ \\
Mean fixation duration in target region & MFD & $262(43)$ & $274(34)$ & & $281(38)$ & $282(45)$ \\
Mean fixation count in target region & MFC & $5.78(2.28)$ & $7.72(2.84)$ & & $3.97(1.81)$ & $5.47(2.14)$ \\
Mean number of entries into target region & MNE & $1.93(0.64)$ & $2.32(0.73)$ & & $1.62(0.52)$ & $2.07(0.66)$ \\
Mean total time in target region & TT & $1522(729)$ & $2065(809)$ & $1040(527)$ & $1450(664)$ \\
\hline
\end{tabular}




\section{Figures captions}

Figure 1. Example of social normal (A), social weird (B), perceptual normal (C) and perceptual weird (D) stimuli. The black square represents the target region. Note the black square is not visible during the experiment.

Figure 2. Interaction for first fixation duration (ms) between group and oddity type. Error bars denote + /$1 \mathrm{SE}$. 
A

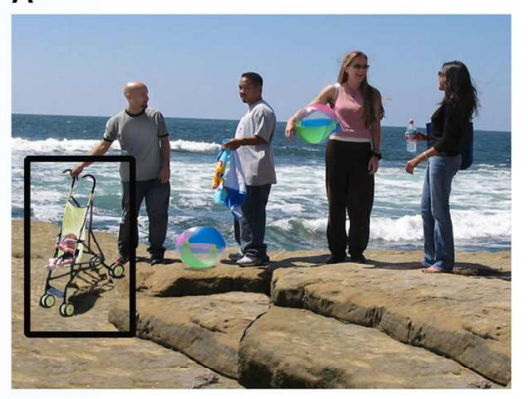

C

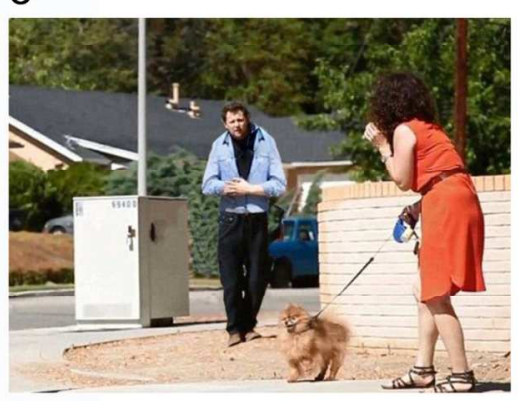

B

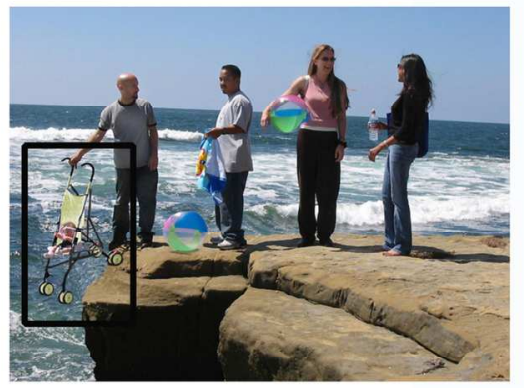

D

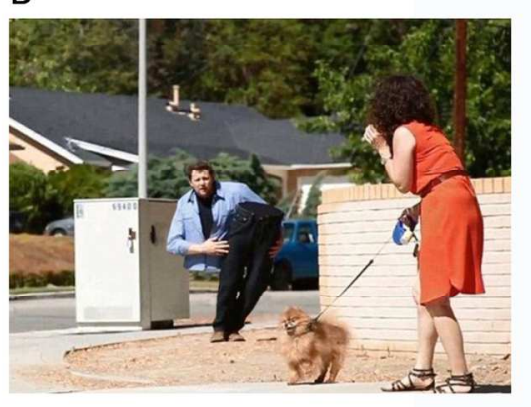

Figure 1. Example of social normal (A), social weird (B), perceptual normal (C) and perceptual weird (D) stimuli. The black square represents the target region. Note the black square is not visible during the experiment.

$149 \times 129 \mathrm{~mm}(300 \times 300 \mathrm{DPI})$ 
1

2

3

4

5

6

7

9

10

11

12

13

14

15

16

17

18

19

20

21

22

23

24

25

26

27

28

29

30

31

32

33

34

35

36

37

38

39

40

41

42

43

44

45

46

47

48

49

50

51

52

53

54

55

56

57

58

59

60

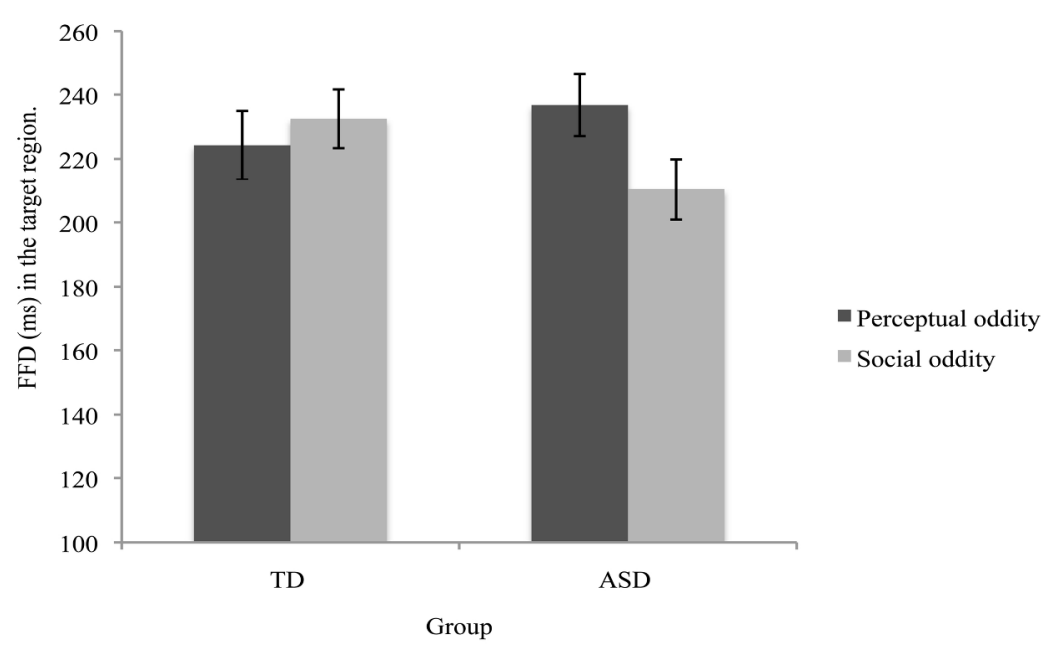

Figure 2. Interaction for first fixation duration (ms) between group and oddity type. Error bars denote +/- 1 $\mathrm{SE}$.

$228 \times 158 \mathrm{~mm}(300 \times 300 \mathrm{DPI})$ 
1

2

3

4

5

6

7

8

9

10

11

12

13

14

15

16

17

18

19

20

21

22

23

24

25

26

27

28

29

30

31

32

33

34

35

36

37

38

39

40

41

42

43

44

45

46

47

48

49

50

51

52

53

54

55

56

57

58

59

60
RUNNING HEAD: LOOKING, SEEING AND BELIEVING IN AUTISM.

Looking, Seeing and Believing in Autism: Eye Movements reveal how subtle

cognitive processing differences impact in the social domain.

Valerie Benson ${ }^{1 *}$, Monica S. Castelhano ${ }^{2}$, Philippa L. Howard ${ }^{1}$, Nida Latif ${ }^{2} \&$ Keith Rayner ${ }^{3}$

AU Psychology, Shackleton Building, Highfield Campus, University of Southampton, UK. 'Queen's University, Kingston, ON, Canada

University of California, San Diego, USA

Telephone: +44 (0) 2380594596 
1

2

3

4

5

6

7

8

9

10

11

12

13

14

15

16

17

18

19

20

21

22

23

24

25

26

27

28

29

30

31

32

33

34

35

36

37

38

39

40

41

42

43

44

45

46

47

48

49

50

51

52

53

54

55

56

57

58

59

60
LOOKING, SEEING AND BELIEVING IN AUTISM

Lay Abstract

Adults with High Functioning Autism (ASD) viewed scenes with people in them, whilst having their eye movements recorded. The task was to indicate, using a button press, whether the pictures were normal, or in some way weird or odd. Oddities in the pictures were categorized as violations of either perceptual or social norms. Compared to a Typically Developed (TD) control group, the ASD participants were equally able to categorise the scenes as odd or normal, but they took longer to respond. The eye movement patterns showed that the ASD group made more fixations and revisits to the target areas in the odd scenes compared to the TD group. Additionally, when the ASD group first fixated the target areas in the scenes, they failed to initially detect the social oddities. These two findings have clear implications for processing difficulties in ASD for the social domain, where it is important to detect social cues on-line, and where there is little opportunity to go back and recheck possible cues in fast dynamic interactions 
LOOKING, SEEING AND BELIEVING IN AUTISM

\section{Scientific Abstrac}

Autistic Spectrum Disorder (ASD) and Typically Developed (TD) adult participants had to decide whether scenes were 'odd', 'unusual' or 'weird'. All scenes contained people, with 'oddness' operationally defined as either perceptual or social. There were no group differences in accuracy, but the ASD group were slower overall to respond manually. Eye movements revealed that the ASD group made more fixations overall and more re-fixations into the target regions of the pictures, for both conditions. Importantly, as indexed by the first fixation duration the ASD group failed to 'pick up' immediately on what was 'odd' for the social violations. The propensity to 'go back' and re-fixate targets, coupled with a failure to initially detect social violations, has obvious significance for ASD in fast dynamic social communication.

Key phrases: Autism Spectrum Disorder, Eye movements, On-line cognitive processing, Social and Perceptual Oddities. 


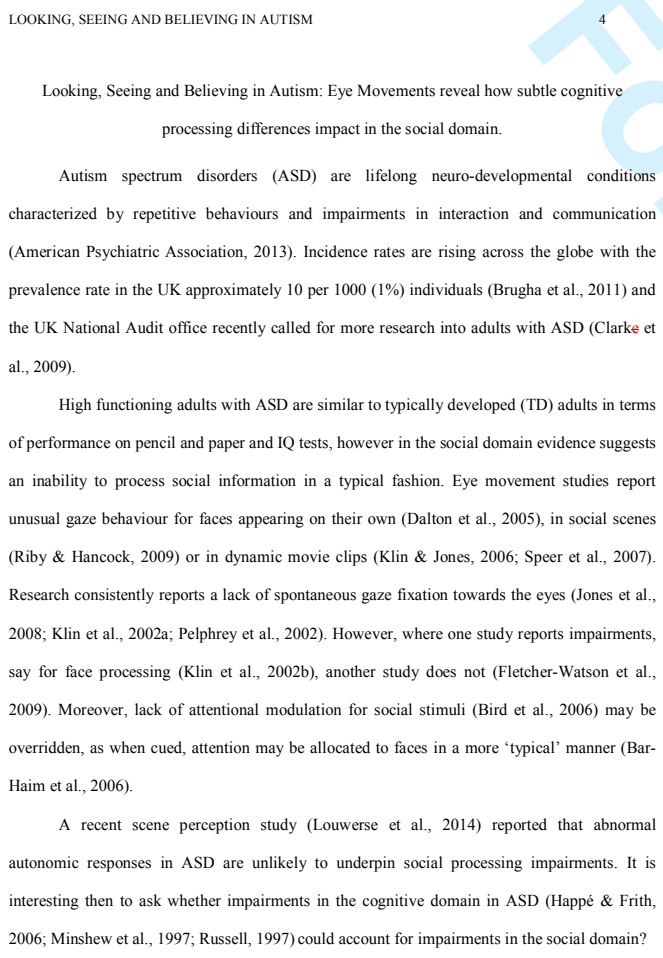




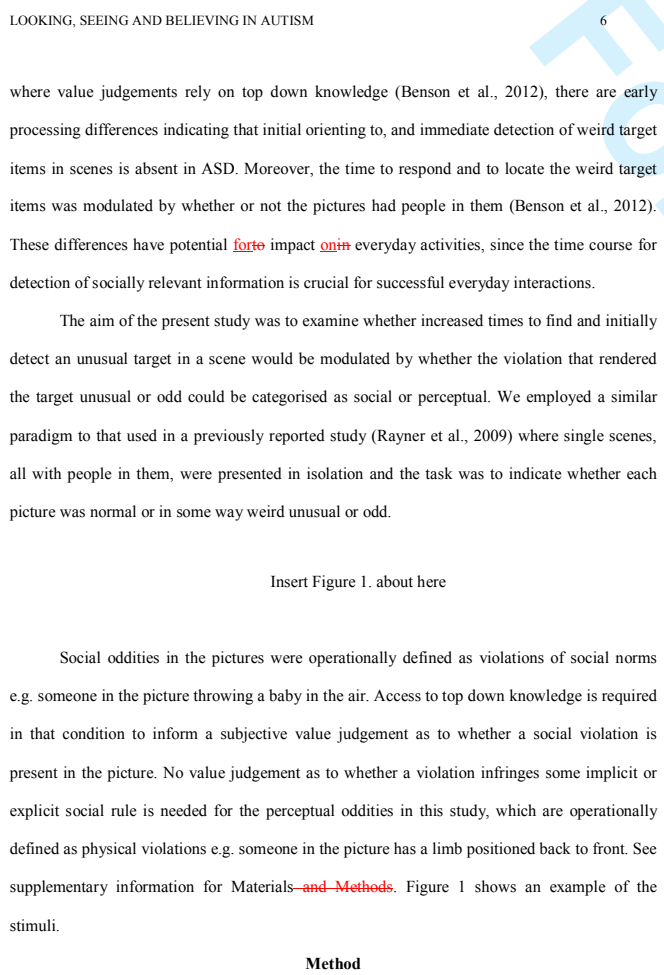




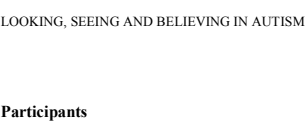


detected automatically using a default velocity criterion of 30 degrees per second using the Eyelink software. See Stampe (1993) for an in depth technical description of how the software detects saccades and fixations. A chin rest and forehead support, were used to stabilize head position. Viewing was binocular but data were analysed for the right eye only. Individual participants were calibrated using a nine point matrix that covered the dimensions of the screen (1024 768 pixels), with each point fixated sequentially, followed by a validation procedure to ensure that fixation was within 0.5 degrees of each calibration point. Calibration was checked using a validation procedure prior to each trial, whereby participants fixated five dots, presented at five different locations that covered the dimensions of the screen and recalibration was performed if the eyes were off centre for any of these dots.

Materials

In total, 96 full-colour photographs were used as stimuli, all of which contained people. In both the perceptual and social oddity conditions there were 48 pictures (24 weird and 24 normal). Perceptual oddities in the pictures violated a physical norm e.g. someone in the picture has a limb positioned back to front, or a vehicle was in an unexpected position e.g midway through the upper wall of a building. Social oddities in the pictures violated a social norm e.g. someone in the picture throwing a baby in the air, or someone with a cabbage on a lead. A small number of the pictures were from a previous study (Benson et al., 2012) and the remainder were collected from the internet and included either a perceptual or social oddity. Adobe Photoshop was used to prepare a normal version of each scene in which the oddity was replaced with either the background elements or with another suitable object. The scenes typically had a focal object or a central group of objects. Figure 1 shows an example of a normal and a socially odd scene, 
Eyelink software was used to detect saccades and fixations using a default velocity criterion and in-house software was used to calculate the distribution of fixations falling into the target region. Five items were removed from analysis because for these scenes, participant accuracy scores were significantly below chance'. In addition, each participant was asked whether they had seen any of the scenes previously, and if so these scenes were also removed. This resulted in a loss of $2.3 \%$ of trials. Fixations below $90 \mathrm{~ms}$ and above $1200 \mathrm{~ms}$ were classed as outliers and removed; resulting in a loss of $5.13 \%$ of the fixation data.

Results

For the principal analyses we compared manual reaction times and accuracy (see Table 1), as well as a number of eye movement measures, such as, the time taken to begin fixating in the target region (ELT), the duration of the first fixation when the eyes landed in the target region (FFD) and the mean time (MFD) spent fixating in the target area (see Table. 2). Global eye movement measures for the target region, such as mean fixation duration and mean number of fixations over all trials were also analysed, Finally, baseline measures (not just target related), were calculated across the duration of the trials, to check for overall sampling differences between the ASD and TD participants (see Table 2). See supplementary information for methods and materials.

\section{Behavioural Data}

Accuracy. There was no effect of oddity type (perceptual vs. social) $F(1,41)=1.44, p=$ $.237, \eta_{\mathrm{p}}{ }^{2}=.03$, picture type (normal or weird) $F(1,41)=0.94 p=.339, \eta_{\mathrm{p}}{ }^{2}=.02$, or group, $F(1$, $41)=.06, p=.808, \eta_{\mathrm{p}}^{2}<.01$ on accuracy rates, and no interactions $\left(F \mathrm{~s}<2.39, p^{\prime} s \geq .130\right)$. At 


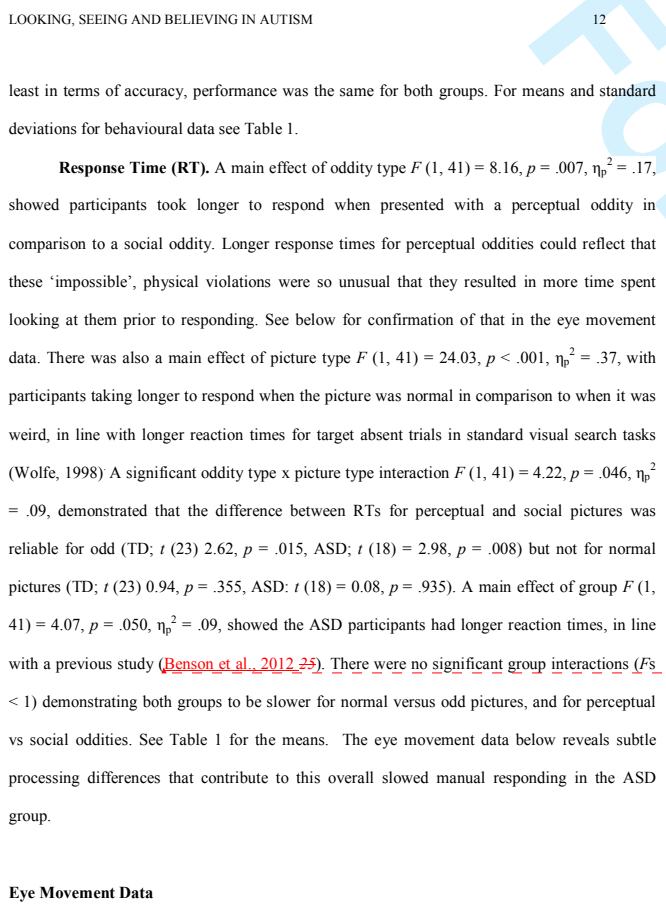


LOOKING, SEEING AND BELIEVING IN AUTISM
Having shown that all participants could distinguish between 'odd' and 'normal' pictures, ormal picture trials were excluded from the eye movement analyses resulting in a 2 (group: ASD versus TD) X 2 (oddity type: social versus perceptual) design to investigate on-line processing differences between the two groups for the two types of oddities.

Baseline eye movement measures, calculated across the full length of trials indicated no group difference in mean fixation duration $F(1,41)=1.14, p=.293, \eta_{\mathrm{p}}{ }^{2}=.03$, but the ASD group made more fixations overall $F(1,41)=9.44, p=.004, \eta_{\mathrm{p}}{ }^{2}=.19$, corresponding to the increased RT's reported above. Table 2 presents a summary of all the eye movement measures.

Elapsed time to the target region (ELT). A marginal effect of oddity type $F(1,41)=3.99, p=$ $.053, \eta_{\mathrm{p}}{ }^{2}=.09$, on the time taken from the onset of each trial display to the start of the first fixation on the target region, revealed both groups took less time to fixate the target region when the oddity was perceptual in comparison to social in nature. The impossible nature of the perceptual violation may have inereased the salience of the target region but-T there was no effect of group $F(1,41)=0.59, p=.446, \eta_{\mathrm{p}}{ }^{2}=.01$, and no interaction $F(1,41)=0.72, p=.401, \eta_{\mathrm{p}}{ }^{2}=$ .02 .

First Fixation Duration (FFD) on target region. There was no effect of oddity type $F(1,41)=$ $1.84, p=.182, \eta_{\mathrm{p}}{ }^{2}=.04$ or group $F(1,41)=0.15, p=.699, \eta_{\mathrm{p}}{ }^{2}<.01$ but a significant interaction $F(1,41)=6.72, p=.013, \eta_{\mathrm{p}}{ }^{2}=.14$ (See Figure 2) revealed that FFDs were equivalent for perceptual and social oddities for TD participants $t(23)=0.86, p=.399, d=.16$, but for ASD participants FFDs for social oddities were significantly shorter, in comparison to perceptual oddities, $t(18)=2.99, p=.008, d=.65$, see Figure 2 . Given that the FFD is a measure of early 
processing _ (Liversedge \& Findlay, 2000; Rayner, 1998; Rayner, 2009)31-33), this finding indicates that the ASD group did not detect the social oddities as quickly as the perceptual oddities on initial fixation, whereas TD participants detected both types of oddity equally fast. Insert Figure 2. about here

First gaze duration (FGD) in the target region. The FGD is the elapsed time from when the target region is first fixated until the eye moves on to a new location. FGD includes multiple fixations in the target region reflects an overt strategy to re-examine an area. A main effect of oddity type, $F(1,41)=12.10, p=.001, \eta_{\mathrm{p}}{ }^{2}=.23$ demonstrated that both groups spent more time inspecting perceptual oddities, again highlighting the salience of the perceptual oddities in comparison to social oddities in this study. However, there was no group effect $F(1,41)=2.43$, $p=.127, \eta_{\mathrm{p}}{ }^{2}=.06$, or interaction $F(1,41)=.08, p=.785, \eta_{\mathrm{p}}{ }^{2}<.01$.

Mean Fixation Count in the target region. If the ASD are slower to respond manually, but do not take longer to begin inspecting the target region, then extra time to respond might reflect more fixations in the target region. A main effect of oddity type $F(1,41)=64.27, p<.001, \eta_{\mathrm{p}}{ }^{2}=$ .61 , demonstrated more fixations in the target region when the oddity was perceptual, rather than social. A main effect of group $F(1,41)=6.99, p=.012, \eta_{\mathrm{p}}{ }^{2}=.15$ showed that the ASD participants-group made more fixations than the TD group in the target regions, for both types of oddities overall. However no interaction was observed $F(1,41)=0.77, p=.386, \eta_{\mathrm{p}}{ }^{2}=.02$ indicating that the ASD group made more fixations in the target regions for both types of oddities.

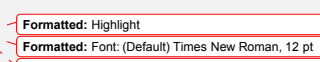

\begin{tabular}{l} 
Formatted: Font: (Default) Times New Roman, $12 \mathrm{pt}$ \\
\hline Formatted: Font: (Default) Times New Roman, $12 \mathrm{pt}$, Not tialic
\end{tabular}

Formatted: Font: Not t talic

Formatted: Font: (Defautt) Times New Roman, $12 \mathrm{pt}$ Formatted: Highlight 


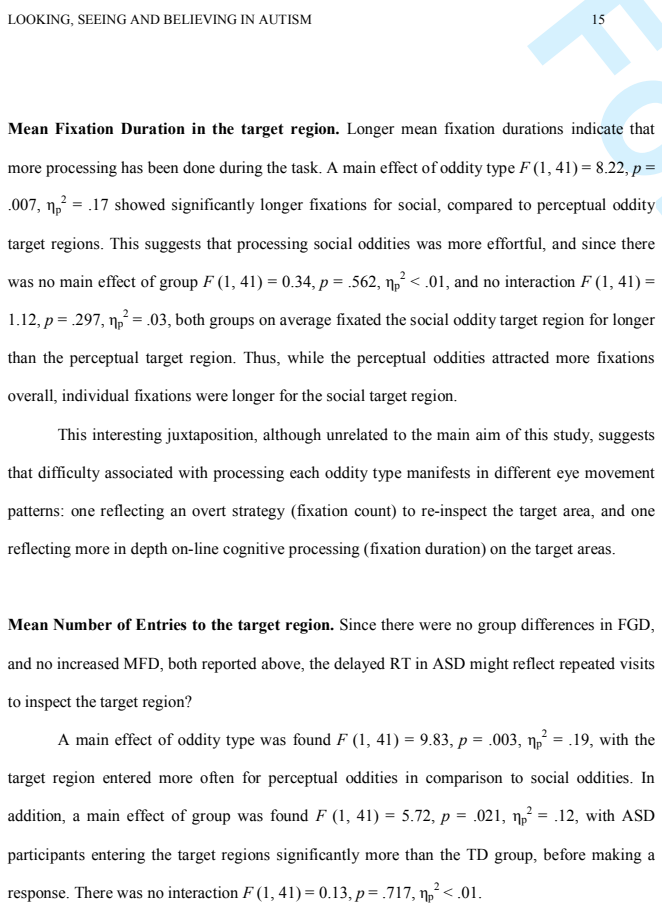


LOOKING, SEEING AND BELIEVING IN AUTISM

Furthermore, these increased fixations were not random, but were made to the target regions of both social and perceptually odd pictures. Thus, increased fixations reflect a propensity to reinspect the target regions prior to making a response in ASD, regardless as to the type of oddity

in the scenes. It should be noted here that the oddity detection task in the current study provides the ASD participants with structure that is not typically available in real-life. As such, the task is not implicit and the knowledge that oddities might be present could also be a factor that drives he repeated scanning in ASD.

The eye movement patterns also showed that our ASD group failed to immediately detect social oddities, thus looking at but not seeing them. An obvious question from this finding is whether the failure to quickly spot subtle social oddities in ASD reflects an inability to react or respond appropriately to social cues in everyday communication? This one impairment in itself could potentially account for the significant problems experienced in dynamic social interactions in this high functioning population. For example, a failure to immediately spot social cues would result in a failure to react or respond to such cues thus resulting in an inability to both keep track of what is going on in social interactions, and a failure to engage appropriately or effectively with other people:-

In relation to current theories that underpin ASD our findings do not fit with WCC theor \& Happé, 1994; Happé \& Frith. 2009), since clearly context can be taken into aceount in ASD during task completion. Moreover, the slowness to respend in ASD is not reflected in longer times to find the target, and the increased fixations in ASD were seen for both perceptulat and social oddities in the scenes. It may be that repeated visits to the target area indicate integration problems in ASD, but even if that were the case, such problems are not restrieted to social processing per se. Neither do the support the executive function theory (Russell, 1997), as Formatted: Font: (Default) Times New Roman, $12 \mathrm{pt}$
Formatted: Font: (Default) Times New Roman, $12 \mathrm{pt}$ Formatted: Font: (Defaut) Times New Roman, 12 pt 
LOOKING, SEEING AND BELIEVING IN AUTISM

ASD participants showed no indication of increased mean fixation durations, thus no evidence of perseveration of any inability to switch foects throughout the task. Our findings could howrever be interpreted to support the EMB (Baron Cohen, 2002), with the detection of social oddities that required empathy being delayed in ASD, however note that higher re inspection of pereeptuat eddities that could be detected on the basis of systematic rules, was also found for ASD participants

If social oddities are more complex to detect in ASD, then the Complex Information Processing theory (Minshew \& Goldstein, 1998) might explain the failure to detect the social oddity on first fixation. This lack of immediate detection could reflect the less obvious and more 'inferential' nature of the social oddities, which required more complex reasoning, at least in the ASD group, for detection. It is important to note that social rules are often implicit, sometimes ambiguous, and cannot always be understood by following a script or flow chart as in the case for logical rule based tasks. At a neurobiological level, it has been postulated that that impairments in cognitive processing in ASD reflect slow information transmission rates for high level cognitive tasks, resulting from underconnectivity between frontal-posterior cortical regions (Just et al., 2013; Just et al., 2012; Minshew \& Williams, 2007). Since underconnectivity reduces the rate of inter-region information transfer, it could be that in social communication tasks that rely on the fast detection of implicit, often ambiguous cues, a failure to be able to transmit information across different cortical areas quickly, might mean that these cues are missed, rendering inappropriate responses in ASD.

The findings of repeated scanning of the target areas, coupled with an inability to immediately recognise social violations upon initial fixation, reflect how eye movements can reveal subtle processing differences in ASD. In everyday social communication in the real world

\section{Formatted: Not Highlight \\ Formatted: Font: (Default) Times New Roman, 12 pt, Not Highligh} Formatted: Not Highlight 
1

2

3

4

5

6

7

8

9

10

11

12

13

14

15

16

17

18

19

20

21

22

23

24

25

26

27

28

29

30

31

32

33

34

35

36

37

38

39

40

41

42

43

44

45

46

47

48

49

50

51

52

53

54

55

56

57

58

59

60
LOOKING, SEEING AND BELIEVING IN AUTISM

there is no opportunity to 'go back' to check if one has missed an important cue. Social processing in such circumstances must be carried out on-line, and our eye movement data clearly show that ASD are unable to do this quickly. The strategy of re-checking their interpretation, and thus not believing what they have seen, before responding, may be related to cortical underconnectivity in ASD. In everyday terms, these findings have obvious consequences for fast dynamic social interactions, and offer an account as to why high functioning adults with ASD experience difficulty in the social domain. 


\section{Page 79 of 116}

Autism Research

1
2
3
4
5
6
7
8
9
10
11
12
13
14
15
16
17
18
19
20
21
22
23
24
25
26
27
28
29
30
31
32
33
34
35
36
37
38
39
40
41
42
43
44
45
50
48
49
50
51
52
54
50

LOOKING, SEEING AND BELIEVING IN AUTISM

Acknowledgments

This work was supported by a grant awarded to the second author from the Natural Sciences and

Engineering Research Council of Canada, the Canadian Foundation for Innovation, the Ontario Ministry of Research and Innovation, and Queen's University Senate Advisory Research Committee, and an Experimental Psychology Society study visit grant awarded to the third author

John Wiley \& Sons 
American Psychiatric Association. (2013). Diagnostic and statistical manual of mental disorders (5th ed.). Arlington, VA: American Psychiatric Publishing.

Au-Yeung, S. K., Benson, V., Castelhano, M. S., Rayner, K. (2011). Eye movement sequences during simple versus complex information processing of scenes in autism spectrum disorder. Autism Research and treatment, 2011.

Au-Yeung, S. K., Kaakinen, J. K., Benson, V. (2013). Cognitive perspective taking during scene perception in autism spectrum disorder: evidence from eye movements. Autism Research, 7, 84-93.

Bar-Haim, Y., Shulman, C., Lamy, D., \& Reuveni, A. (2006). Attention to eyes and mouth in high-functioning children with autism, Journal of Autism and Developmental Disorders, 36, 131-137.

Baron-Cohen, S. (2002). The extreme male brain theory of autism. Trends in Cognitive Science, 6, 248-254.

Baron-Cohen, S., Wheelwright, S., Skinner, R., Martin, J., \& Clubley, E. (2001). The AutismSpectrum Quotient (AQ): Evidence from Asperger syndrome/high-functioning autism, males and females, scientists and mathematicians. Journal of Autism and Developmental Disorders, 31(1), 5-17

Benson, V., \& Fletcher-Watson, S. (2012). Eye Movements in Autism. In: Liversedge, S. P.,

Gilchrist, I. D., Everling, S., editors. The Oxford Handbook of Eye Movements, Oxford UK: Oxford University Press, chap 39 


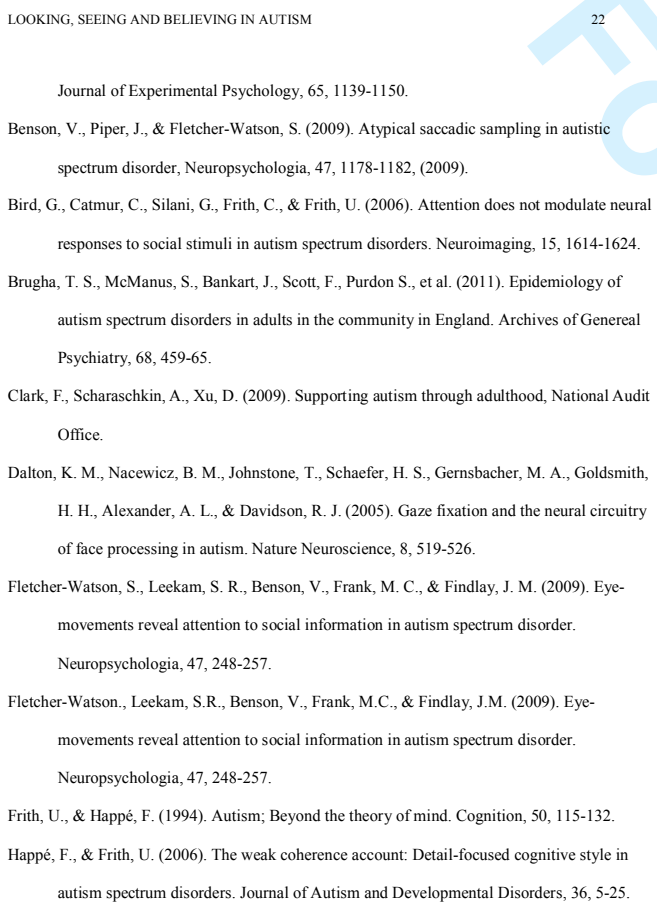




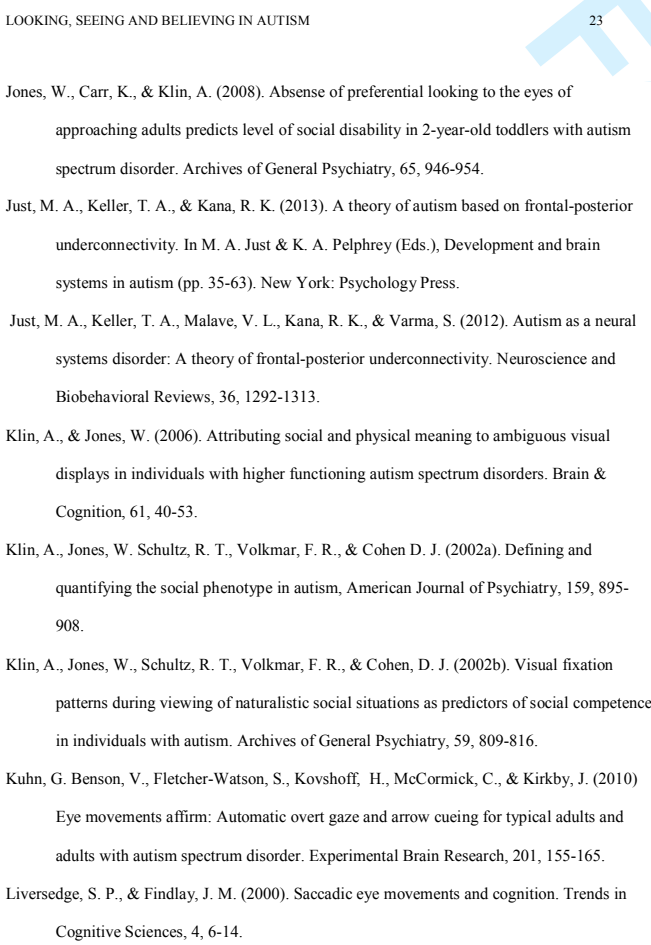




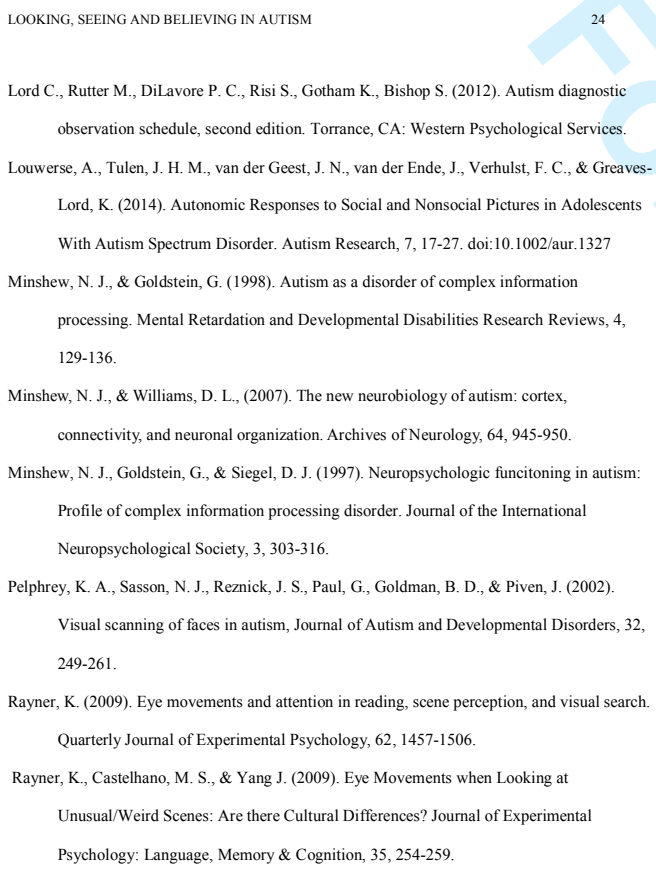




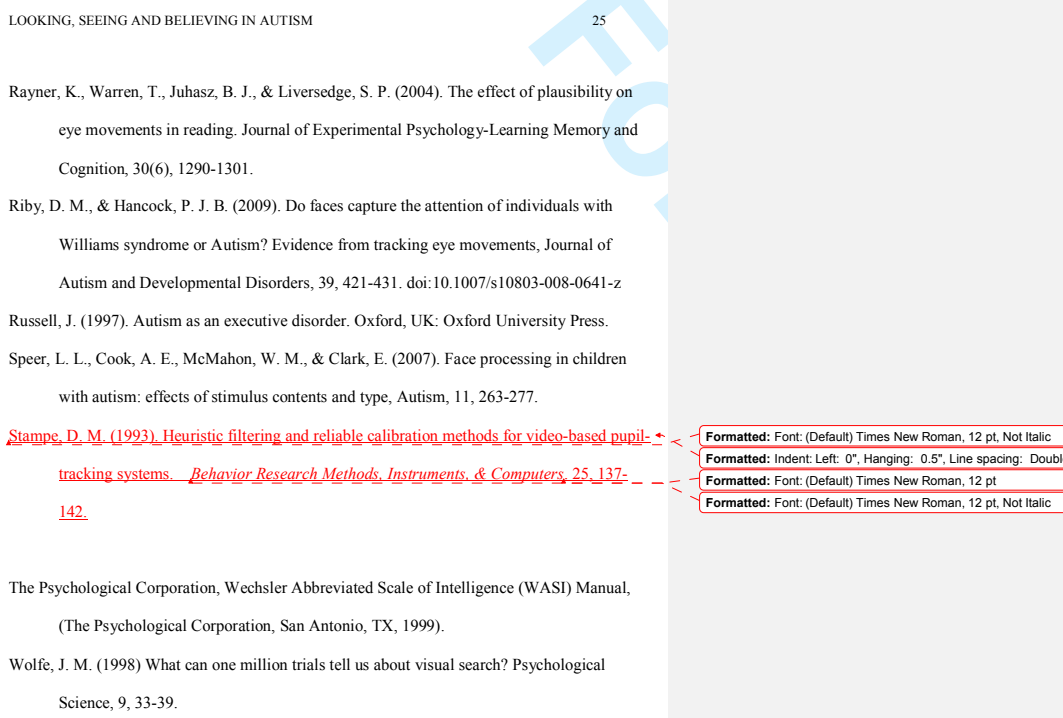




\section{Page 85 of 116}

Autism Research

1
2
3
4
5
6
7
8
9
10
11
12
13
14
15
16
17
18
19
20
21
22
23
24
25
26
27
28
29
30
31
32
33
34
35
36
37
38
39
40
41
42
43
44
45
59
48
49
50
51
53
54
50

LOOKING, SEEING AND BELIEVING IN AUTISM

Footnotes

1. Accuracy errors for the excluded scenes were either a result of a failure to detect a subtle

oddity, or were a result of participants thinking the control version of the scenes were odd.

Removing these items from analysis did not change the pattern of effects, but reduced variation

in reaction time and eye movement data.

John Wiley \& Sons 
32

33

34

35

36

37

38

39

40

41

42

43

44

45

46

47

48

49

50

51

52

53

54

55

56

57

58

59

60

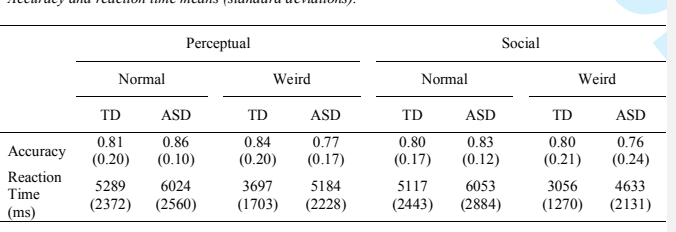


Table 2.

\begin{tabular}{|c|c|c|c|c|c|}
\hline \multicolumn{4}{|c|}{ RUNNING HEAD: LOOKING, SEEING AND BELIEVING IN AUTISM. } & \multicolumn{2}{|l|}{28} \\
\hline \multicolumn{6}{|l|}{ Table 2.} \\
\hline \multicolumn{6}{|c|}{ Eye movement measure means in ms (standard deviations). } \\
\hline & & \multicolumn{2}{|c|}{ Perceptual Oddity } & \multicolumn{2}{|c|}{ Social Oddity } \\
\hline & & TD & ASD & TD & ASD \\
\hline Mean Fixation Duration (overall) & & $250(31)$ & $257(29)$ & $241(26)$ & $252(33)$ \\
\hline Mean Fixation Count (overall) & & $11(4)$ & $15(6)$ & $9(4)$ & $13(7)$ \\
\hline Elapsed time to target region & ELT & $551(235)$ & $557(144)$ & $600(235)$ & $679(284)$ \\
\hline Fixation count before target region & FCT & $2.32(0.72)$ & $2.49(0.67)$ & $2.62(0.76)$ & $2.68(0.73)$ \\
\hline First fixation duration in target region & FFD & $224(52)$ & $237(42)$ & $232(45)$ & $210(41)$ \\
\hline First gaze duration in target region & FGD & $808(250)$ & $924(437)$ & $630(163)$ & $715(208)$ \\
\hline Mean fixation duration in target region & MFD & $262(43)$ & $274(34)$ & $281(38)$ & $282(45)$ \\
\hline Mean fixation count in target region & MFC & $5.78(2.28)$ & $7.72(2.84)$ & $3.97(1.81)$ & $5.47(2.14)$ \\
\hline Mean number of entries into target region & MNE & $1.93(0.64)$ & $2.32(0.73)$ & $1.62(0.52)$ & $2.07(0.66)$ \\
\hline Mean total time in target region & тт & $1522(729)$ & $2065(809)$ & $1040(527)$ & $1450(664)$ \\
\hline
\end{tabular}


1

2

3

4

5

6

7

8

9

10

11

12

13

14

15

16

17

18

19

20

21

22

23

24

25

26

27

28

29

30

31

32

33

34

35

36

37

38

39

40

41

42

43

44

45

46

47

48

49

50

51

52

53

54

55

56

57

58

59

60
RUNNING HEAD: LOOKING, SEEING AND BELIEVING IN AUTISM.

Figures captions

tigure 1. Example of social normal (A), social weird (B), perceptual normal (C) and perceptual weird (D)

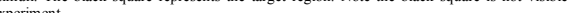

Figure 2. Interaction for first fixation duration (ms) between group and oddity type. Error bars denote $+\ell-$ 
Looking, Seeing and Believing in Autism: Eye Movements reveal how subtle cognitive processing differences impact in the social domain.

\author{
Valerie Benson ${ }^{1 *}$, Monica S. Castelhano ${ }^{2}$, Philippa L. Howard ${ }^{1}$, Nida Latif $^{2}$ \& Keith Rayner $^{3}$ \\ ${ }^{1}$ AU Psychology, Shackleton Building, Highfield Campus, University of Southampton, UK. \\ ${ }^{2}$ Queen's University, Kingston, ON, Canada \\ ${ }^{3}$ University of California, San Diego, USA \\ *Correspondence to: vb1@soton.ac.uk \\ Telephone: +44 (0) 2380594596
}




\section{Lay Abstract}

Adults with High Functioning Autism (ASD) viewed scenes with people in them, whilst having their eye movements recorded. The task was to indicate, using a button press, whether the pictures were normal, or in some way weird or odd. Oddities in the pictures were categorized as violations of either perceptual or social norms. Compared to a Typically Developed (TD) control group, the ASD participants were equally able to categorise the scenes as odd or normal, but they took longer to respond. The eye movement patterns showed that the ASD group made more fixations and revisits to the target areas in the odd scenes compared to the TD group. Additionally, when the ASD group first fixated the target areas in the scenes, they failed to initially detect the social oddities. These two findings have clear implications for processing difficulties in ASD for the social domain, where it is important to detect social cues on-line, and where there is little opportunity to go back and recheck possible cues in fast dynamic interactions. 
Scientific Abstract

\begin{abstract}
Autistic Spectrum Disorder (ASD) and Typically Developed (TD) adult participants had to decide whether scenes were 'odd', 'unusual' or 'weird'. All scenes contained people, with 'oddness' operationally defined as either perceptual or social. There were no group differences in accuracy, but the ASD group were slower overall to respond manually. Eye movements revealed that the ASD group made more fixations overall and more re-fixations into the target regions of the pictures, for both conditions. Importantly, as indexed by the first fixation duration the ASD group failed to 'pick up' immediately on what was 'odd' for the social violations. The propensity to 'go back' and re-fixate targets, coupled with a failure to initially detect social violations, has obvious significance for ASD in fast dynamic social communication.
\end{abstract}

Key phrases: Autism Spectrum Disorder, Eye movements, On-line cognitive processing, Social and Perceptual Oddities. 
Looking, Seeing and Believing in Autism: Eye Movements reveal how subtle cognitive processing differences impact in the social domain.

Autism spectrum disorders (ASD) are lifelong neuro-developmental conditions characterized by repetitive behaviours and impairments in interaction and communication (American Psychiatric Association, 2013). Incidence rates are rising across the globe with the prevalence rate in the UK approximately 10 per 1000 (1\%) individuals (Brugha et al., 2011) and the UK National Audit office recently called for more research into adults with ASD (Clark et al., 2009).

High functioning adults with ASD are similar to typically developed (TD) adults in terms of performance on pencil and paper and IQ tests, however in the social domain evidence suggests an inability to process social information in a typical fashion. Eye movement studies report unusual gaze behaviour for faces appearing on their own (Dalton et al., 2005), in social scenes (Riby \& Hancock, 2009) or in dynamic movie clips (Klin \& Jones, 2006; Speer et al., 2007). Research consistently reports a lack of spontaneous gaze fixation towards the eyes (Jones et al., 2008; Klin et al., 2002a; Pelphrey et al., 2002). However, where one study reports impairments, say for face processing (Klin et al., 2002b), another study does not (Fletcher-Watson et al., 2009). Moreover, lack of attentional modulation for social stimuli (Bird et al., 2006) may be overridden, as when cued, attention may be allocated to faces in a more 'typical' manner (BarHaim et al., 2006).

A recent scene perception study (Louwerse et al., 2014) reported that abnormal autonomic responses in ASD are unlikely to underpin social processing impairments. It is interesting then to ask whether impairments in the cognitive domain in ASD (Happé \& Frith, 2006; Minshew et al., 1997; Russell, 1997) could account for impairments in the social domain? 
Current cognitive theories of ASD include, among others, Weak Central Coherence (WCC) theory (Frith \& Happé, 1994; Happé \& Frith, 2006) which proposes that a reduced sensitivity to global context in favour of focussing on details, results in integration difficulties in ASD. Executive function theory (Russell, 1997), proposes that ASD are impaired at switching their focus of attention, and may engage in perseverative behaviour. Alternatively, the Complex Information Processing theory (Minshew \& Goldstein, 1998) proposes that individuals with ASD have impairments in processing complex information with intact processing for tasks that require simple (rule based) processing. Finally, the Extreme Male Brain (EMB) theory (Baron-Cohen, 2002) posits that individuals with ASD have above average levels of systemizing, that contribute to the non-social characteristics of the disorder, coupled with below average levels of empathizing, which results in atypical social functioning. These contemporary theories of ASD can, for different reasons, offer some account of ineffective social functioning in ASD. However, to date very few investigations have examined online processing differences in ASD.

Our studies investigating cognitive processing in adults with ASD (Au-Yeung et al., 2011; Au-Yeung et al., 2013; Benson et al., 2012; Benson et al., 2009; Benson \& FletcherWatson, 2011) have found subtle on-line processing differences, as revealed by eye movements, for a range of tasks that are more in line with Minshew and Goldstein's (1998) theory, than with any other. For example, there are more similarities than differences in eye movements between TD and ASD groups for simple rule based tasks in the attentional (Kuhn et al., 2010) and the social domains (Benson \& Fletcher-Watson, 2011). Whereas for more complex abstract reasoning tasks where there is ambiguity with relation to target items (Au-Yeung et al., 2013), or where value judgements rely on top down knowledge (Benson et al., 2012), there are early processing differences indicating that initial orienting to, and immediate detection of weird target 
items in scenes is absent in ASD. Moreover, the time to respond and to locate the weird target items was modulated by whether or not the pictures had people in them (Benson et al., 2012). These differences have potential for impact on everyday activities, since the time course for detection of socially relevant information is crucial for successful everyday interactions.

The aim of the present study was to examine whether increased times to find and initially detect an unusual target in a scene would be modulated by whether the violation that rendered the target unusual or odd could be categorised as social or perceptual. We employed a similar paradigm to that used in a previously reported study (Rayner et al., 2009) where single scenes, all with people in them, were presented in isolation and the task was to indicate whether each picture was normal or in some way weird unusual or odd.

Insert Figure 1. about here

Social oddities in the pictures were operationally defined as violations of social norms e.g. someone in the picture throwing a baby in the air. Access to top down knowledge is required in that condition to inform a subjective value judgement as to whether a social violation is present in the picture. No value judgement as to whether a violation infringes some implicit or explicit social rule is needed for the perceptual oddities in this study, which are operationally defined as physical violations e.g. someone in the picture has a limb positioned back to front. See supplementary information for Materials. Figure 1 shows an example of the stimuli.

\section{Method}

\section{Participants}

ASD participants ( $n=24$ age $18-52$ years, 20 males), with a formal diagnosis of either Autistic Disorder $(n=3)$ or Asperger's Syndrome were recruited from local charitable 
organisations. TD participants $(n=24$, age $20-52$ years, 20 males) were volunteers from the local community. All participants were paid and had normal or corrected to normal vision. Each ASD participant completed module 4 of the Autism Diagnostic Observation Scale 2 (Lord et al., 2012) and five (3 males and 2 females with a diagnosis of Asperger's Syndrome) failed to meet the Autism Spectrum criteria. To ensure maximum homogeneity in our study those five participants were excluded from the analyses, resulting in a final sample of 19 ASD participants. Both groups completed the Autism-spectrum Quotient (AQ; Baron-Cohen et al., 2001) and the ASD participants reported a higher number of autistic traits $t(41)=8.04, p<.001$ (TD $M=$ $15.42, S D=7.55 ;$ ASD $M=35.32, S D=8.45)$. The two groups did not differ in age $t(41)=$ $0.31, p=.759(\mathrm{TD} M=30.29, S D=10.20 ;$ ASD $M=31.26, S D=10.29)$, performance IQ $t(41)$ $=0.21, p=.832(\mathrm{TD} M=111.79, S D=12.01 ; \mathrm{ASD} M=110.95, S D=13.84)$, verbal IQ $t(41)=$ $1.21, p=.234(\mathrm{TD} M=116.96, S D=10.65, \mathrm{ASD} M=112.26, S D=14.84)$, or full scale IQ $t$ $(46)=0.87, p=.392(\operatorname{TD} M=116.04, S D=9.98$, ASD $M=112.95, S D=13.48)$, as measured by two performance (Block Design and Matrix Reasoning) and verbal components (Vocabulary and Similarities) of the Weschler Abbrevated Scale of Intelligence (The Psychological Corporation, 1999).

\section{Eye movement recording}

Eye movements were sampled at $1000 \mathrm{~Hz}$ using an Eyelink tracker with spatial accuracy of less than $1 / 4^{\text {th }}$ degree (S.R. Research Ltd, Canada). The start and end of saccades were detected automatically using a default velocity criterion of 30degrees per second using the Eyelink software. See Stampe (1993) for an in depth technical description of how the software detects saccades and fixations. A chin rest and forehead support, were used to stabilize head 
position. Viewing was binocular but data were analysed for the right eye only. Individual participants were calibrated using a nine point matrix that covered the dimensions of the screen (1024 x 768 pixels), with each point fixated sequentially, followed by a validation procedure to ensure that fixation was within 0.5 degrees of each calibration point. Calibration was checked using a validation procedure prior to each trial, whereby participants fixated five dots, presented at five different locations that covered the dimensions of the screen and recalibration was performed if the eyes were off centre for any of these dots.

\section{Materials}

In total, 96 full-colour photographs were used as stimuli, all of which contained people. In both the perceptual and social oddity conditions there were 48 pictures (24 weird and 24 normal). Perceptual oddities in the pictures violated a physical norm e.g. someone in the picture has a limb positioned back to front, or a vehicle was in an unexpected position e.g midway through the upper wall of a building. Social oddities in the pictures violated a social norm e.g. someone in the picture throwing a baby in the air, or someone with a cabbage on a lead. A small number of the pictures were from a previous study (Benson et al., 2012) and the remainder were collected from the internet and included either a perceptual or social oddity. Adobe Photoshop was used to prepare a normal version of each scene in which the oddity was replaced with either the background elements or with another suitable object. The scenes typically had a focal object or a central group of objects. Figure 1 shows an example of a normal and a socially odd scene, and below those examples, a normal and a perceptually odd scene. The target regions for these scenes are outlined in black - but these black outlines were not visible during the presentation of the scenes in the experiment. 
A norming study was conducted to verify the validity of the images used to examine the processing of social and perceptual violations. Twenty undergraduates from Queen's university participated ( $M$ age: 19.8years, $S D: 1.71,10$ Females) with five participants in each of the four conditions (counterbalanced conditions: weird/normal and right/left button response). Stimuli were displayed on a 2 inch CRT monitor with a refresh rate of $100 \mathrm{~Hz}$ and the experiment was run using E-Prime software. Participants were presented with a fixation cross for $500 \mathrm{~ms}$ followed by the presentation of the image for 20 seconds or until a keyboard "Y' (yes) or "N" (N) was made. Participants had to indicate as quickly as possible whether the presented image was "odd", "weird" or "unusual" in some way. The proportion of identified images indicated that on average, the social normal $(M=0.86, S D=0.15)$ and social weird $(M=0.84, S D=0.17)$, perceptual normal $(M=0.82, S D=0.17)$ and perceptual weird $(M=0.77, S D=0.19)$ images were identified correctly.

\section{Design}

For the behavioural measures of Accuracy and RT a mixed design with Picture Type (weird versus normal) and Oddity Type (social versus perceptual) as within participants variables, and Group (ASD versus TD) as a between participants variable was employed for the experiment. On condition that accuracy was equivalent for both normal and weird pictures, for the eye movement measures the main focus of interest was to compare social versus perceptual oddities in both groups. Therefore a mixed design with Oddity Type (social versus perceptual) as a within participants variable, and Group (ASD versus TD) as a between participants variable was employed in the eye movement analysis. 


\section{Procedure}

All participants were shown a hard copy of an example picture from each condition prior to completing the task, to ensure comprehension of the task and the ability to respond appropriately. The specific instruction was 'for each of the following pictures, please indicate whether you think the picture is odd, unusual or strange in some way'.

For the task, 48 pictures were presented randomly to either the left or the right of the centre of a 19inch colour monitor, at a distance of $65 \mathrm{~cm}$ for $20 \mathrm{~s}$ or until a button press response was made. Prior to the onset of a picture, participants fixated a cross that was presented on the opposite side of the screen to where the picture was set to appear, to ensure all participants started at the same point in each trial. Once participants fixated this cross, the experimenter initiated each trial. Picture type (normal vs. weird) and, the position of the picture (left or right on the display) were counterbalanced across items. The buttons used to respond either yes or no (left or right trigger button) was counterbalanced across participants.

\section{Data preparation and analyses}

For each 'odd' picture we created an interest region around the target item and the corresponding region of the same size in the counterpart 'normal' picture. Regions of interest were created using the approximate average regions from those identified and manually selected by participants who took part in the norming experiment.

Eyelink software was used to detect saccades and fixations using a default velocity criterion and in-house software was used to calculate the distribution of fixations falling into the target region. Five items were removed from analysis because for these scenes, participant accuracy scores were significantly below chance ${ }^{1}$. In addition, each participant was asked whether they had seen 
any of the scenes previously, and if so these scenes were also removed. This resulted in a loss of $2.3 \%$ of trials. Fixations below $90 \mathrm{~ms}$ and above $1200 \mathrm{~ms}$ were classed as outliers and removed; resulting in a loss of $5.13 \%$ of the fixation data.

\section{Results}

For the principal analyses we compared manual reaction times and accuracy (see Table 1), as well as a number of eye movement measures, such as, the time taken to begin fixating in the target region (ELT), the duration of the first fixation when the eyes landed in the target region (FFD) and the mean time (MFD) spent fixating in the target area (see Table. 2). Global eye movement measures for the target region, such as mean fixation duration and mean number of fixations over all trials were also analysed, Finally, baseline measures (not just target related), were calculated across the duration of the trials, to check for overall sampling differences between the ASD and TD participants (see Table 2). See supplementary information for methods and materials.

\section{Behavioural Data}

Accuracy. There was no effect of oddity type (perceptual vs. social) $F(1,41)=1.44, p=$ $.237, \eta_{\mathrm{p}}{ }^{2}=.03$, picture type (normal or weird) $F(1,41)=0.94 p=.339, \eta_{\mathrm{p}}{ }^{2}=.02$, or group, $F(1$, $41)=.06, p=.808, \eta_{\mathrm{p}}{ }^{2}<.01$ on accuracy rates, and no interactions $\left(F_{\mathrm{S}}<2.39, p\right.$ 's $\left.>.130\right)$. At least in terms of accuracy, performance was the same for both groups. For means and standard deviations for behavioural data see Table 1.

Response Time (RT). A main effect of oddity type $F(1,41)=8.16, p=.007, \eta_{\mathrm{p}}{ }^{2}=.17$, showed participants took longer to respond when presented with a perceptual oddity in 
comparison to a social oddity. Longer response times for perceptual oddities could reflect that these 'impossible', physical violations were so unusual that they resulted in more time spent looking at them prior to responding. See below for confirmation of that in the eye movement data. There was also a main effect of picture type $F(1,41)=24.03, p<.001, \eta_{\mathrm{p}}{ }^{2}=.37$, with participants taking longer to respond when the picture was normal in comparison to when it was weird, in line with longer reaction times for target absent trials in standard visual search tasks (Wolfe, 1998) A significant oddity type x picture type interaction $F(1,41)=4.22, p=.046, \eta_{\mathrm{p}}{ }^{2}$ $=.09$, demonstrated that the difference between RTs for perceptual and social pictures was reliable for odd (TD; $t$ (23) 2.62, $p=.015$, ASD; $t(18)=2.98, p=.008$ ) but not for normal pictures $(\mathrm{TD} ; t(23) 0.94, p=.355$, ASD: $t(18)=0.08, p=.935)$. A main effect of group $F(1$, $41)=4.07, p=.050, \eta_{\mathrm{p}}{ }^{2}=.09$, showed the ASD participants had longer reaction times, in line with a previous study (Benson et al., 2012 ). There were no significant group interactions $(F \mathrm{~s}<$ 1) demonstrating both groups to be slower for normal versus odd pictures, and for perceptual vs social oddities. See Table 1 for the means. The eye movement data below reveals subtle processing differences that contribute to this overall slowed manual responding in the ASD group.

\section{Eye Movement Data}

Having shown that all participants could distinguish between 'odd' and 'normal' pictures, normal picture trials were excluded from the eye movement analyses resulting in a 2 (group: ASD versus TD) X 2 (oddity type: social versus perceptual) design to investigate on-line processing differences between the two groups for the two types of oddities. 
Baseline eye movement measures, calculated across the full length of trials indicated no group difference in mean fixation duration $F(1,41)=1.14, p=.293, \eta_{\mathrm{p}}{ }^{2}=.03$, but the ASD group made more fixations overall $F(1,41)=9.44, p=.004, \eta_{\mathrm{p}}{ }^{2}=.19$, corresponding to the increased RT's reported above. Table 2 presents a summary of all the eye movement measures.

Elapsed time to the target region (ELT). A marginal effect of oddity type $F(1,41)=3.99, p=$ $.053, \eta_{\mathrm{p}}{ }^{2}=.09$, on the time taken from the onset of each trial display to the start of the first fixation on the target region, revealed both groups took less time to fixate the target region when the oddity was perceptual in comparison to social in nature. There was no effect of group $F$ (1, $41)=0.59, p=.446, \eta_{\mathrm{p}}{ }^{2}=.01$, and no interaction $F(1,41)=0.72, p=.401, \eta_{\mathrm{p}}{ }^{2}=.02$.

First Fixation Duration (FFD) on target region. There was no effect of oddity type $F(1,41)=$ $1.84, p=.182, \eta_{\mathrm{p}}{ }^{2}=.04$ or group $F(1,41)=0.15, p=.699, \eta_{\mathrm{p}}{ }^{2}<.01$ but a significant interaction $F(1,41)=6.72, p=.013, \eta_{\mathrm{p}}{ }^{2}=.14$ (See Figure 2) revealed that FFDs were equivalent for perceptual and social oddities for TD participants $t(23)=0.86, p=.399, d=.16$, but for ASD participants FFDs for social oddities were significantly shorter, in comparison to perceptual oddities, $t(18)=2.99, p=.008, d=.65$, see Figure 2 . Given that the FFD is a measure of early processing (Liversedge \& Findlay, 2000; Rayner, 1998; Rayner, 2009). this finding indicates that the ASD group did not detect the social oddities as quickly as the perceptual oddities on initial fixation, whereas TD participants detected both types of oddity equally fast.

Insert Figure 2. about here 
First gaze duration (FGD) in the target region. The FGD is the elapsed time from when the target region is first fixated until the eye moves on to a new location. FGD includes multiple fixations in the target region reflects an overt strategy to re-examine an area. A main effect of oddity type, $F(1,41)=12.10, p=.001, \eta_{\mathrm{p}}{ }^{2}=.23$ demonstrated that both groups spent more time inspecting perceptual oddities, again highlighting the salience of the perceptual oddities in comparison to social oddities in this study. However, there was no group effect $F(1,41)=2.43$, $p=.127, \eta_{\mathrm{p}}{ }^{2}=.06$, or interaction $F(1,41)=.08, p=.785, \eta_{\mathrm{p}}{ }^{2}<.01$.

Mean Fixation Count in the target region. If the ASD are slower to respond manually, but do not take longer to begin inspecting the target region, then extra time to respond might reflect more fixations in the target region. A main effect of oddity type $F(1,41)=64.27, p<.001, \eta_{\mathrm{p}}{ }^{2}=$ .61 , demonstrated more fixations in the target region when the oddity was perceptual, rather than social. A main effect of group $F(1,41)=6.99, p=.012, \eta_{\mathrm{p}}{ }^{2}=.15$ showed that the ASD group made more fixations than the TD group in the target regions, for both types of oddities. However no interaction was observed $F(1,41)=0.77, p=.386, \eta_{\mathrm{p}}{ }^{2}=.02$ indicating that the ASD group made more fixations in the target regions for both types of oddities.

Mean Fixation Duration in the target region. Longer mean fixation durations indicate that more processing has been done during the task. A main effect of oddity type $F(1,41)=8.22, p=$ $.007, \eta_{\mathrm{p}}{ }^{2}=.17$ showed significantly longer fixations for social, compared to perceptual oddity target regions. This suggests that processing social oddities was more effortful, and since there was no main effect of group $F(1,41)=0.34, p=.562, \eta_{\mathrm{p}}{ }^{2}<.01$, and no interaction $F(1,41)=$ $1.12, p=.297, \eta_{\mathrm{p}}{ }^{2}=.03$, both groups on average fixated the social oddity target region for longer 
than the perceptual target region. Thus, while the perceptual oddities attracted more fixations overall, individual fixations were longer for the social target region.

This interesting juxtaposition, although unrelated to the main aim of this study, suggests that difficulty associated with processing each oddity type manifests in different eye movement patterns: one reflecting an overt strategy (fixation count) to re-inspect the target area, and one reflecting more in depth on-line cognitive processing (fixation duration) on the target areas.

Mean Number of Entries to the target region. Since there were no group differences in FGD, and no increased MFD, both reported above, the delayed RT in ASD might reflect repeated visits to inspect the target region?

A main effect of oddity type was found $F(1,41)=9.83, p=.003, \eta_{\mathrm{p}}{ }^{2}=.19$, with the target region entered more often for perceptual oddities in comparison to social oddities. In addition, a main effect of group was found $F(1,41)=5.72, p=.021, \eta_{\mathrm{p}}{ }^{2}=.12$, with ASD participants entering the target regions significantly more than the TD group, before making a response. There was no interaction $F(1,41)=0.13, p=.717, \eta_{\mathrm{p}}^{2}<.01$.

Mean Total Time in the target region. A higher mean total time means that more attention to, and processing of, information in that region was carried out overall. This measure should complement the measures reported above - namely, that if there are more fixations overall in ASD then the Total Time spent fixating in the target region should be longer in ASD.

A main effect of oddity type $F(1,41)=34.89, p<.001, \eta_{\mathrm{p}}{ }^{2}=.46$, showed perceptual oddities received more overall processing time, in line with the previous measures for the perceptual oddities. In addition, a main effect of group $F(1,41)=6.41, p=.015, \eta_{\mathrm{p}}{ }^{2}=.14$ 
revealed that the ASD participants spent a longer period of time inspecting the target regions for both perceptual and social oddities. There was no interaction $F(1,41)=0.51, p=.481, \eta_{\mathrm{p}}{ }^{2}=.01$.

As above, this finding reflects a greater number of entries to the target region overall by the ASD group prior to making their decision as to whether the picture under inspection was weird, odd or unusual, irrespective of the nature of the violation (perceptual or social).

\section{Discussion}

A principal aim of the study was to investigate whether increased times in ASD to find and initially detect an unusual target in a scene containing people would be modulated by whether the violation could be categorised as social or perceptual.

Accuracy rates were equivalent and high for both groups, but the ASD group took longer to respond manually. It was hypothesised that the eye movement data would reveal the cause of this delayed response in ASD, since eye movements reflect how information is processed on-line (Liversedge \& Findlay, 2000; Rayner, 1998; 2009). The data showed that our ASD group did not take longer to find the target regions, but that they scanned the scenes more prior to responding manually, as shown by an increased number of fixations throughout each scene presentation. Furthermore, these increased fixations were not random, but were made to the target regions of both social and perceptually odd pictures. Thus, increased fixations reflect a propensity to reinspect the target regions prior to making a response in ASD, regardless as to the type of oddity in the scenes. It should be noted here that the oddity detection task in the current study provides the ASD participants with structure that is not typically available in real-life. As such, the task is not implicit and the knowledge that oddities might be present could also be a factor that drives the repeated scanning in ASD. 
The eye movement patterns also showed that our ASD group failed to immediately detect social oddities, thus looking at but not seeing them. An obvious question from this finding is whether the failure to quickly spot subtle social oddities in ASD reflects an inability to react or respond appropriately to social cues in everyday communication? This one impairment in itself could potentially account for the significant problems experienced in dynamic social interactions in this high functioning population. For example, a failure to immediately spot social cues would result in a failure to react or respond to such cues thus resulting in an inability to both keep track of what is going on in social interactions, and a failure to engage appropriately or effectively with other people.

If social oddities are more complex to detect in ASD, then the Complex Information Processing theory (Minshew \& Goldstein, 1998) might explain the failure to detect the social oddity on first fixation. This lack of immediate detection could reflect the less obvious and more 'inferential' nature of the social oddities, which required more complex reasoning, at least in the ASD group, for detection. It is important to note that social rules are often implicit, sometimes ambiguous, and cannot always be understood by following a script or flow chart as in the case for logical rule based tasks. At a neurobiological level, it has been postulated that that impairments in cognitive processing in ASD reflect slow information transmission rates for high level cognitive tasks, resulting from underconnectivity between frontal-posterior cortical regions (Just et al., 2013; Just et al., 2012; Minshew \& Williams, 2007). Since underconnectivity reduces the rate of inter-region information transfer, it could be that in social communication tasks that rely on the fast detection of implicit, often ambiguous cues, a failure to be able to transmit information across different cortical areas quickly, might mean that these cues are missed, rendering inappropriate responses in ASD. 
The findings of repeated scanning of the target areas, coupled with an inability to immediately recognise social violations upon initial fixation, reflect how eye movements can reveal subtle processing differences in ASD. In everyday social communication in the real world there is no opportunity to 'go back' to check if one has missed an important cue. Social processing in such circumstances must be carried out on-line, and our eye movement data clearly show that ASD are unable to do this quickly. The strategy of re-checking their interpretation, and thus not believing what they have seen, before responding, may be related to cortical underconnectivity in ASD. In everyday terms, these findings have obvious consequences for fast dynamic social interactions, and offer an account as to why high functioning adults with ASD experience difficulty in the social domain. 


\section{Acknowledgments}

This work was supported by a grant awarded to the second author from the Natural Sciences and Engineering Research Council of Canada, the Canadian Foundation for Innovation, the Ontario Ministry of Research and Innovation, and Queen's University Senate Advisory Research Committee, and an Experimental Psychology Society study visit grant awarded to the third author. 


\section{References}

American Psychiatric Association. (2013). Diagnostic and statistical manual of mental disorders (5th ed.). Arlington, VA: American Psychiatric Publishing.

Au-Yeung, S. K., Benson, V., Castelhano, M. S., Rayner, K. (2011). Eye movement sequences during simple versus complex information processing of scenes in autism spectrum disorder. Autism Research and treatment, 2011.

Au-Yeung, S. K., Kaakinen, J. K., Benson, V. (2013). Cognitive perspective taking during scene perception in autism spectrum disorder: evidence from eye movements. Autism Research, 7, 84-93.

Bar-Haim, Y., Shulman, C., Lamy, D., \& Reuveni, A. (2006). Attention to eyes and mouth in high-functioning children with autism, Journal of Autism and Developmental Disorders, $36,131-137$.

Baron-Cohen, S. (2002). The extreme male brain theory of autism. Trends in Cognitive Science, $6,248-254$.

Baron-Cohen, S., Wheelwright, S., Skinner, R., Martin, J., \& Clubley, E. (2001). The AutismSpectrum Quotient (AQ): Evidence from Asperger syndrome/high-functioning autism, males and females, scientists and mathematicians. Journal of Autism and Developmental Disorders, 31(1), 5-17.

Benson, V., \& Fletcher-Watson, S. (2012). Eye Movements in Autism. In: Liversedge, S. P., Gilchrist, I. D., Everling, S., editors. The Oxford Handbook of Eye Movements, Oxford UK: Oxford University Press, chap 39.

Benson, V., Castelhano, M. S., Au-Yeung, S. K., \& Rayner K. (2012). Eye movements reveal no immediate "WOW" ("which one's weird") effect in autism spectrum disorder, Quarterly 
Journal of Experimental Psychology, 65, 1139-1150.

Benson, V., Piper, J., \& Fletcher-Watson, S. (2009). Atypical saccadic sampling in autistic spectrum disorder, Neuropsychologia, 47, 1178-1182, (2009).

Bird, G., Catmur, C., Silani, G., Frith, C., \& Frith, U. (2006). Attention does not modulate neural responses to social stimuli in autism spectrum disorders. Neuroimaging, 15, 1614-1624.

Brugha, T. S., McManus, S., Bankart, J., Scott, F., Purdon S., et al. (2011). Epidemiology of autism spectrum disorders in adults in the community in England. Archives of Genereal Psychiatry, 68, 459-65.

Clark, F., Scharaschkin, A., Xu, D. (2009). Supporting autism through adulthood, National Audit Office.

Dalton, K. M., Nacewicz, B. M., Johnstone, T., Schaefer, H. S., Gernsbacher, M. A., Goldsmith, H. H., Alexander, A. L., \& Davidson, R. J. (2005). Gaze fixation and the neural circuitry of face processing in autism. Nature Neuroscience, 8, 519-526.

Fletcher-Watson, S., Leekam, S. R., Benson, V., Frank, M. C., \& Findlay, J. M. (2009). Eyemovements reveal attention to social information in autism spectrum disorder. Neuropsychologia, 47, 248-257.

Fletcher-Watson., Leekam, S.R., Benson, V., Frank, M.C., \& Findlay, J.M. (2009). Eyemovements reveal attention to social information in autism spectrum disorder. Neuropsychologia, 47, 248-257.

Frith, U., \& Happé, F. (1994). Autism; Beyond the theory of mind. Cognition, 50, 115-132.

Happé, F., \& Frith, U. (2006). The weak coherence account: Detail-focused cognitive style in autism spectrum disorders. Journal of Autism and Developmental Disorders, 36, 5-25. 
Jones, W., Carr, K., \& Klin, A. (2008). Absense of preferential looking to the eyes of approaching adults predicts level of social disability in 2-year-old toddlers with autism spectrum disorder. Archives of General Psychiatry, 65, 946-954.

Just, M. A., Keller, T. A., \& Kana, R. K. (2013). A theory of autism based on frontal-posterior underconnectivity. In M. A. Just \& K. A. Pelphrey (Eds.), Development and brain systems in autism (pp. 35-63). New York: Psychology Press.

Just, M. A., Keller, T. A., Malave, V. L., Kana, R. K., \& Varma, S. (2012). Autism as a neural systems disorder: A theory of frontal-posterior underconnectivity. Neuroscience and Biobehavioral Reviews, 36, 1292-1313.

Klin, A., \& Jones, W. (2006). Attributing social and physical meaning to ambiguous visual displays in individuals with higher functioning autism spectrum disorders. Brain \& Cognition, 61, 40-53.

Klin, A., Jones, W. Schultz, R. T., Volkmar, F. R., \& Cohen D. J. (2002a). Defining and quantifying the social phenotype in autism, American Journal of Psychiatry, 159, 895908.

Klin, A., Jones, W., Schultz, R. T., Volkmar, F. R., \& Cohen, D. J. (2002b). Visual fixation patterns during viewing of naturalistic social situations as predictors of social competence in individuals with autism. Archives of General Psychiatry, 59, 809-816.

Kuhn, G. Benson, V., Fletcher-Watson, S., Kovshoff, H., McCormick, C., \& Kirkby, J. (2010) Eye movements affirm: Automatic overt gaze and arrow cueing for typical adults and adults with autism spectrum disorder. Experimental Brain Research, 201, 155-165.

Liversedge, S. P., \& Findlay, J. M. (2000). Saccadic eye movements and cognition. Trends in Cognitive Sciences, 4, 6-14. 
Lord C., Rutter M., DiLavore P. C., Risi S., Gotham K., Bishop S. (2012). Autism diagnostic observation schedule, second edition. Torrance, CA: Western Psychological Services.

Louwerse, A., Tulen, J. H. M., van der Geest, J. N., van der Ende, J., Verhulst, F. C., \& GreavesLord, K. (2014). Autonomic Responses to Social and Nonsocial Pictures in Adolescents With Autism Spectrum Disorder. Autism Research, 7, 17-27. doi:10.1002/aur.1327

Minshew, N. J., \& Goldstein, G. (1998). Autism as a disorder of complex information processing. Mental Retardation and Developmental Disabilities Research Reviews, 4, 129-136.

Minshew, N. J., \& Williams, D. L., (2007). The new neurobiology of autism: cortex, connectivity, and neuronal organization. Archives of Neurology, 64, 945-950.

Minshew, N. J., Goldstein, G., \& Siegel, D. J. (1997). Neuropsychologic funcitoning in autism: Profile of complex information processing disorder. Journal of the International Neuropsychological Society, 3, 303-316.

Pelphrey, K. A., Sasson, N. J., Reznick, J. S., Paul, G., Goldman, B. D., \& Piven, J. (2002). Visual scanning of faces in autism, Journal of Autism and Developmental Disorders, 32, 249-261.

Rayner, K. (2009). Eye movements and attention in reading, scene perception, and visual search. Quarterly Journal of Experimental Psychology, 62, 1457-1506.

Rayner, K., Castelhano, M. S., \& Yang J. (2009). Eye Movements when Looking at Unusual/Weird Scenes: Are there Cultural Differences? Journal of Experimental Psychology: Language, Memory \& Cognition, 35, 254-259. 
Rayner, K., Warren, T., Juhasz, B. J., \& Liversedge, S. P. (2004). The effect of plausibility on eye movements in reading. Journal of Experimental Psychology-Learning Memory and Cognition, 30(6), 1290-1301.

Riby, D. M., \& Hancock, P. J. B. (2009). Do faces capture the attention of individuals with Williams syndrome or Autism? Evidence from tracking eye movements, Journal of Autism and Developmental Disorders, 39, 421-431. doi:10.1007/s10803-008-0641-z

Russell, J. (1997). Autism as an executive disorder. Oxford, UK: Oxford University Press.

Speer, L. L., Cook, A. E., McMahon, W. M., \& Clark, E. (2007). Face processing in children with autism: effects of stimulus contents and type, Autism, 11, 263-277.

Stampe, D. M. (1993). Heuristic filtering and reliable calibration methods for video-based pupiltracking systems. Behavior Research Methods, Instruments, \& Computers. 25, $137-$ 142.

The Psychological Corporation, Wechsler Abbreviated Scale of Intelligence (WASI) Manual, (The Psychological Corporation, San Antonio, TX, 1999).

Wolfe, J. M. (1998) What can one million trials tell us about visual search? Psychological Science, 9, 33-39. 


\section{Footnotes}

1. Accuracy errors for the excluded scenes were either a result of a failure to detect a subtle oddity, or were a result of participants thinking the control version of the scenes were odd.

Removing these items from analysis did not change the pattern of effects, but reduced variation in reaction time and eye movement data. 


\section{Tables}

Table 1.

Accuracy and reaction time means (standard deviations).

\begin{tabular}{|c|c|c|c|c|c|c|c|c|}
\hline & \multicolumn{4}{|c|}{ Perceptual } & \multicolumn{4}{|c|}{ Social } \\
\hline & \multicolumn{2}{|c|}{ Normal } & \multicolumn{2}{|c|}{ Weird } & \multicolumn{2}{|c|}{ Normal } & \multicolumn{2}{|c|}{ Weird } \\
\hline & TD & ASD & TD & ASD & TD & ASD & TD & ASD \\
\hline Accuracy & $\begin{array}{c}0.81 \\
(0.20)\end{array}$ & $\begin{array}{c}0.86 \\
(0.10)\end{array}$ & $\begin{array}{c}0.84 \\
(0.20)\end{array}$ & $\begin{array}{c}0.77 \\
(0.17)\end{array}$ & $\begin{array}{c}0.80 \\
(0.17)\end{array}$ & $\begin{array}{c}0.83 \\
(0.12)\end{array}$ & $\begin{array}{c}0.80 \\
(0.21)\end{array}$ & $\begin{array}{c}0.76 \\
(0.24)\end{array}$ \\
\hline $\begin{array}{l}\text { Reaction } \\
\text { Time } \\
(\mathrm{ms})\end{array}$ & $\begin{array}{c}5289 \\
(2372)\end{array}$ & $\begin{array}{c}6024 \\
(2560)\end{array}$ & $\begin{array}{c}3697 \\
(1703)\end{array}$ & $\begin{array}{c}5184 \\
(2228)\end{array}$ & $\begin{array}{c}5117 \\
(2443)\end{array}$ & $\begin{array}{c}6053 \\
(2884)\end{array}$ & $\begin{array}{c}3056 \\
(1270)\end{array}$ & $\begin{array}{c}4633 \\
(2131)\end{array}$ \\
\hline
\end{tabular}


Table 2 .

Eye movement measure means in ms (standard deviations).

\begin{tabular}{lcccccc}
\hline & & \multicolumn{2}{c}{ Perceptual Oddity } & & Social Oddity \\
\cline { 3 - 5 } \cline { 5 - 6 } & & TD & ASD & & TD & ASD \\
\hline Mean Fixation Duration (overall) & & $250(31)$ & $257(29)$ & & $241(26)$ & $252(33)$ \\
Mean Fixation Count (overall) & & $11(4)$ & $15(6)$ & & $9(4)$ & $13(7)$ \\
Elapsed time to target region & ELT & $551(235)$ & $557(144)$ & & $600(235)$ & $679(284)$ \\
Fixation count before target region & FCT & $2.32(0.72)$ & $2.49(0.67)$ & & $2.62(0.76)$ & $2.68(0.73)$ \\
First fixation duration in target region & FFD & $224(52)$ & $237(42)$ & & $232(45)$ & $210(41)$ \\
First gaze duration in target region & FGD & $808(250)$ & $924(437)$ & & $630(163)$ & $715(208)$ \\
Mean fixation duration in target region & MFD & $262(43)$ & $274(34)$ & & $281(38)$ & $282(45)$ \\
Mean fixation count in target region & MFC & $5.78(2.28)$ & $7.72(2.84)$ & & $3.97(1.81)$ & $5.47(2.14)$ \\
Mean number of entries into target region & MNE & $1.93(0.64)$ & $2.32(0.73)$ & & $1.62(0.52)$ & $2.07(0.66)$
\end{tabular}




\section{Figures captions}

Figure 1. Example of social normal (A), social weird (B), perceptual normal (C) and perceptual weird (D) stimuli. The black square represents the target region. Note the black square is not visible during the experiment.

Figure 2. Interaction for first fixation duration (ms) between group and oddity type. Error bars denote + /$1 \mathrm{SE}$. 